\title{
Optical and radio survey of southern compact groups of galaxies
}

\section{Pilot study of six groups ${ }^{\star, \star \star}$}

\author{
E. Pompei ${ }^{1}$, M. Dahlem ${ }^{2}$, and A. Iovino ${ }^{3}$ \\ 1 European Southern Observatory (ESO), Alonso de Cordova 3107, Santiago, Chile \\ e-mail: epompei@eso.org \\ 2 CSIRO/ATNF Paul Wild Observatory, Locked Bag 194, Narrabri NSW 2390, Australia \\ 3 Osservatorio Astronomico di Brera, via Brera 28, Milan, Italy
}

Received 21 January 2007 / Accepted 19 July 2007

\section{ABSTRACT}

\begin{abstract}
Context. Multi-wavelength observations of Hickson's Compact Groups (HCGs) have shown that many of these groups are physical bound structures and are in different stage of evolution, from spiral-dominated systems to almost merged objects. Very few studies have analysed the Southern Compact Groups (SCGs) sample, which is thought to be younger that HCGs, due to an on average higher number of spiral galaxies. We present here the first results from optical and radio observations on a pilot sample of SCGs.

Aims. Previous HI studies of HCGs seems to corroborate an evolutionary sequence from low velocity dispersion, spiral dominated, young and star-forming groups to higher velocity dispersion, mostly early-type, X-ray bright groups. In this scheme, the dominant parameter is most likely the ratio between the galaxy mass vs. the available gas mass. A second parameter, namely the merging history of the group, however might have an important role in the evolution of the intra-group medium. Powerful merging events and/or interactions can contribute significantly to the heating of the intra-group medium by means of supernova explosions or by triggering an AGN. We propose to investigate whether the evolutionary scheme of the intragroup medium found for HCGs also holds in a different compact group sample. In addition to this, we start in this paper to investigate which is the influence of the merging history of groups on their evolution.

Methods. Optical observations of SCGs obtained with ESO telescopes and radio data from the ATCA allow us to probe the distribution of the warm intra-group medium and the evolutionary stage of each group, by means of morphological studies and via measurements of star formation and other types of nuclear activity.

Results. We present here results from a pilot sample of SCGs: we find an evolutionary trend, based on the properties of the warm intra-group medium, similar to what has been found for HCGs. Both galaxies with Sy2 nuclei in our sample are members of groups in the late stages of their dynamical evolution. However while one group is strongly HI deficient and shows, at the same time a strong ongoing merging, the other does not show any HI deficiency and the galaxies are only interacting with each other.
\end{abstract}

Key words. galaxies: interactions - galaxies: fundamental parameters - galaxies: general - galaxies: intergalactic medium

\section{Introduction}

Compact groups of galaxies are among the densest and smallest associations of galaxies which can be found on the sky. Comprising 4 to 10 galaxies, with a galaxy-galaxy separation comparable to the individual galaxy diameters and a velocity dispersion of the order of $200 \mathrm{~km} \mathrm{~s}^{-1}$, they are the best-suited laboratories for studies of strong galaxy interactions; even more so in the absence of massive elliptical galaxies and their associated gravitationally heated X-ray-emitting intergalactic envelopes, as found in massive clusters.

However strong mergings in the Hickson's compact group sample are rare, of the order of $6-7 \%$ of the total number of galaxies (Mendes de Oliveira et al. 1994). Tidal interactions, on the other hand, occur frequently, with $50 \%$ of the galaxies in Hickson's sample showing signs of morphological disturbances.

The interactions often involve more than two galaxies, which leads to a large number of targets for investigations of the effects of tidal forces on the member galaxies.

* Figures 1,3-12 and Table 3 are only available in electronic form at http://www. aanda.org

$\star \star$ Tables 8 and 9 are only available in electronic form at the CDS via anonymous ftp to cdsarc.u-strasbg.fr (130.79.128.5) or via http://cdsweb.u-strasbg.fr/cgi-bin/qcat?J/A+A/473/399
In such close proximity it is also likely that tidal interactions, galaxy harassment and similar processes play a major role in the evolution of the galaxies and of their morphological type. Hickson's groups show a remarkable lack of spiral galaxies with respect to field galaxies, $49 \%$ against $82 \%$, and approximately $2 / 3$ of the groups show diffuse X-ray intra-group emission (Ponman et al. 1996). While some groups, like HCG 16 (Ribeiro et al. 1998), are still very active and rich in spirals, most of the HCGs appear to be in an advanced stage of evolution, rich in early type galaxies and surrounded by an hot intragroup medium. The warm intragroup medium seems to follow an evolutionary sequence driven mainly by the ratio of galaxy mass vs gas mass (Verdes-Montenegro et al. 2001, herafter VM01): from H I mainly centered on member galaxies to groups without any warm gas at all or to groups with diffuse H I often offset with respect to the stellar components of the member galaxies.

The motivation for the work presented here is to investigate how groups richer in late type galaxies will evolve and what is the fate of their warm gas.

The Southern Compact Group sample (hereafter SCGs), described in detail by Iovino (2002, hereafter I02), is richer in spirals than HCGs. In fact, $\sim 70 \%$ of its member galaxies are spirals.

The catalog, based on the COSMOS scans of the SERC $(J)$ southern sky survey, has been created following criteria very 
Table 1. Salient parameters of six Southern Compact Groups of galaxies. $N_{\text {conc }}$ is the number of concordant galaxies in the original SCGs catalog, while $N_{\text {Gal }}$ is the total number of confirmed member galaxies fulfilling the group selection criteria and having a similar redshift from optical and radio data.

\begin{tabular}{lcccccc}
\hline \hline Group name & Alternate name & $\begin{array}{c}\text { Centre Position }^{a} \\
\alpha, \delta(2000)\end{array}$ & $\begin{array}{c}v_{\text {hel }}^{b} \\
\left(\mathrm{~km} \mathrm{~s}^{-1}\right)\end{array}$ & $\begin{array}{c}D^{c} \\
(\mathrm{Mpc})\end{array}$ & $N_{\text {conc }}$ & $N_{\text {Gal }}$ \\
\hline SCG 2159-3210 & HCG90 & $22: 02: 02.6,-31: 55: 26$ & 2643 & 35.5 & 4 & 4 \\
SCG 2353-6101 & - & $23: 55: 47.0,-60: 43: 06$ & 4538 & 61.2 & 3 & 3 \\
SCG 0018-4854 & AM0018-485 & $00: 21: 23.1,-48: 38: 19$ & 3386 & 45.5 & 4 & 5 \\
SCG 0122-3819 & - & $01: 25: 16.0,-38: 08: 35$ & 6125 & 82.9 & 4 & 5 \\
SCG 0141-3429 & USCG S063 & $01: 43: 24.0,-34: 14: 42$ & 3905 & 52.6 & 5 & 5 \\
SCG 0227-4212 & - & $02: 29: 30.9,-43: 01: 41$ & 5384 & 72.8 & 6 & 6 \\
\hline
\end{tabular}

${ }^{a}$ Approximate centre positions; pointing centres of radio observations. ${ }^{b}$ Mean value of all measured optical recession velocities. ${ }^{c}$ Based on $H_{0}=67 \mathrm{~km} \mathrm{~s}^{-1} \mathrm{Mpc}^{-1}, \Omega_{\mathrm{m}}=0.3$ and $\Omega_{\Lambda}=0.7$.

similar to the Hickson's ones, but allowing for faint galaxies in the so called isolation ring, thus avoiding one of the biases of Hickson's catalog, i.e. to discard groups whose fainter galaxy members had a magnitude close to the cut-off limit. For more details, we refer the reader to the cited work, however, for completeness sake, we report here the selection criteria:

- Richness: $n \geq 4$, where $n$ is the number of galaxies within $\Delta \operatorname{mag}_{\text {comp }} \leq 3$, where $\Delta$ mag $_{\text {comp }}$ is the magnitude difference between the brightest group member ( $\left.m_{\text {brightest }}\right)$ and the faintest $\left(m_{\text {faintest }}\right)$.

- Isolation: $R_{\text {isolation }} \geq 3 \times R_{\text {group }}$ where $R_{\text {group }}$ is the radius of the smallest circle containing the centres of all group members and $R_{\text {isolation }}$ is the distance from the centre of the circle to the nearest non-member galaxy with a magnitude $m>m_{\text {faintest }}+0.35 \mathrm{mag}$.

- Compactness: $\mu_{\mathrm{gr}} \leq \mu_{\text {limit }}$, where $\mu_{\mathrm{gr}}$ is the mean surface brightness within the circle of radius $R_{\text {group }}$ and $\mu_{\text {limit }}=27.7$ in the $b_{j}$ band.

A bright subsample of the SCGs, i.e. all those groups whose brightest galaxy has $b_{j} \leq 14.5$, for a total of 50 groups, has been extensively observed in the optical: results on the star formation history and galaxy activity in SCGs have been presented by Coziol et al. (2000). At the moment, however, very little information is available on the SCGs intragroup medium, either warm or hot: this means that we lack an important diagnostic of the evolutionary history of the groups and of the interactions among member galaxies.

To this aim, we have started a multi-wavelength observing campaign, including optical imaging and spectroscopy, as well as radio observations. Here we present, as a first showcase, optical, H I and radio continuum data of a small number of objects, for which good-quality data are available already.

Optical imaging in the $R$ filter traces the stellar distributions and provides information on morphology; spectroscopy, through measurements of abundances and star formation rates, will tell us whether galaxies have predominantly old or young stellar populations.

$\mathrm{H} \mathrm{I}$ is the best tracer of gas being pulled out of galaxies taking part in gravitational interactions.

Radio continuum and $\mathrm{H} \alpha$ trace star formation (SF) triggered by radial flows in the disturbed gravitational potential of the group members.

Star formation in three SCGs, including SCG 0018-4854, is described briefly by Temporin et al. (2005).

This paper is divided as follows: in Sect. 2 we describe the optical and radio observations and data reduction, in Sect. 3 we present our results, which are discussed in Sect. 4. Section 5 contains our conclusions.

Throughout the paper, a $\Lambda$ CDM cosmology $\left(\Omega_{\mathrm{M}}=0.3\right.$; $\left.\Omega_{\Lambda}=0.7\right)$ and $\mathrm{H}_{0}=67 \mathrm{~km} \mathrm{~s}^{-1} \mathrm{Mpc}^{-1}$ have been used.

\section{Observations and data reduction}

The targets studied by us were selected from the brighter subsample of the Southern Compact Groups Survey (I02), based on target visibility during the allocated observing periods. All targets, with the exception of SCG2159-3210, are spiral dominated groups, and they represent a fair pilot sample of the SCGs survey. Their main characteristics are given in Table 1.

\subsection{Optical data}

Optical images and spectra of the groups presented here have been obtained at La Silla Observatory during different runs. We discuss below the main results on six groups obtained from these data, while we defer the full presentation of the optical data to a future paper.

\subsubsection{Optical broad-band imaging}

Optical images of a large number of SCGs have been obtained at the Danish $1.54 \mathrm{~m}$ telescope, with the Danish Faint Object Spectrograph and Camera (DFOSC) in the $R$ filter during two separate runs, in August 1995 and August 1996. The data presented in this paper are part of the 1996 run only; during this year the DFOSC CCD was upgraded to a $2048 \times 2048$ pixels array, with a pixel size of $0.39^{\prime \prime} /$ pix and a field of view of $14^{\prime} \times 14^{\prime}$; the seeing during the observations varied between $1.0^{\prime \prime}$ and $1.4^{\prime \prime}$. In this paper we present also additional data, again with the $R$ filter, acquired in January 2006 using Eso Faint Object Spectrograph and Camera 2 (EFOSC2) on the $3.6 \mathrm{~m}$ telescope. The $2048 \times$ 2048 pixels CCD has a field of view of $5.4^{\prime} \times 5.4^{\prime}$ and a pixel scale of $0.157^{\prime \prime} /$ pixel and it was used in binned mode; average seeing on the images was $0.9^{\prime \prime}$.

Data reduction has been carried out with $\mathrm{IRAF}^{1}$ in the standard way; photometric zero points have been measured from Landolt fields observed during the same nights of the observations. A $\log$ of the observations and the zero-points is shown in Table 2.

The GALFIT software (Peng et al. 2002) was used to fit the surface brightness distribution of the member galaxies of

\footnotetext{
1 Image Reduction and Analysis Facility, written and supported by the IRAF programming group at the National Optical Astronomy Observatories (NOAO).
} 
Table 2. Observing log; SCG 2159-3210 has not been observed by us in the optical and related results are taken from the literature.

\begin{tabular}{|c|c|c|c|c|c|c|}
\hline Group & Date of observations & Telescope and instrument & Filter & $\begin{array}{l}\text { Pixel scale } \\
\text { (arcsec) }\end{array}$ & $\begin{array}{c}\text { Exposure time } \\
\text { (s) }\end{array}$ & Zero-Point \\
\hline SCG 2353-6101 & August 1996 & Danish $1.54 \mathrm{~m}+\mathrm{DFOSC}$ & ESO\#452 (R) & 0.39 & $3 \times 200$ & $23.92 \pm 0.03$ \\
\hline SCG 0018-4854 & August 1996 & Danish $1.54 \mathrm{~m}+$ DFOSC & ESO\#452 (R) & 0.39 & $3 \times 200$ & $23.92 \pm 0.03$ \\
\hline SCG 0122-3819 & August 1996 & Danish $1.54 \mathrm{~m}+\mathrm{DFOSC}$ & ESO\#452 (R) & 0.39 & $3 \times 200$ & $23.92 \pm 0.03$ \\
\hline SCG 0141-3429 & January 2006 & $3.6 \mathrm{~m}$ telescope $+\mathrm{EFOSC} 2$ & ESO\#642 (R) & 0.32 & $5 \times 90$ & $26.20 \pm 0.02$ \\
\hline SCG 0227-4212 & August 1996 & Danish $1.54 \mathrm{~m}+\mathrm{DFOSC}$ & ESO\#452 (R) & 0.39 & $3 \times 200$ & $23.92 \pm 0.03$ \\
\hline
\end{tabular}

each group and identifying the different components (bulge, bar, disk and central source) and their respective brightness and scale length. The isophotes could be reliably traced down to 25.4 mag in $R$, and results for all galaxies are shown in Table 3 at the end of the paper. Postage-stamp images of the galaxies, the models obtained from the software and the residuals are shown in Fig. 1. Residual maps from the fitting are used to identify small scale structures, which would otherwise have remained undetected on the original images.

\subsubsection{Optical spectroscopy}

The complete brighter subsample of the SCGs has been observed at the ESO $1.52 \mathrm{~m}$ telescope equipped with the Boller \& Chivens spectrograph in several runs from 1995 to 1997. Instrument set-up, data reduction and analysis, as well as the complete spectroscopic classification of the groups, with the exception of SCG0141-3429, can be found in Coziol et al. (2000).

More sensitive spectroscopic observations have been obtained at the $3.6 \mathrm{~m}$ telescope with the EFOSC2 spectro-imager between 1999 and 2006, including also fainter groups from the SCGs catalog. The increased depth of these data and the improved spatial sampling allow for a more precise spectroscopic classification.

More detailed spectroscopic results for studies of the chemical evolution of galaxies and star formation history in compact groups will be presented elsewhere, while here we use optical spectroscopic results to measure recession velocities, and thereby establish group membership of galaxies, and to classify which type of nuclear activity is observed in the member galaxies of each group.

Observations of SCG0141-3429 are presented here for the first time; this is due to the fact that the magnitude of its brightest galaxy is 14.54 , which led to discard this group from the brightest SCGs subsample. The data were obtained in 2006 with the $3.6 \mathrm{~m}$ telescope and EFOSC2, equipped with grism \#11 and $1^{\prime \prime}$ slit, reading the CCD in binned mode. Standard data reduction was carried on using MIDAS $^{2}$ and a dedicated set of scripts; unfortunately the conditions of the night were not optimal (thin clouds and airmass ranging from 1.2 to 1.9), and no flux calibration was possible. The spectra however are of sufficient quality to allow a preliminary classification of the galaxies activity based on line ratios.

\subsection{ATCA radio data}

Data of six SCGs were obtained with the Australia Telescope Compact Array $\left(\mathrm{ATCA}^{3}\right.$ ) in 1995, a short summary of which was given by Oosterloo \& Iovino (1997). We have now retrieved

${ }^{2}$ Munich Image Data Analysis System, developed and maintained by European Southern Observatory (ESO).

3 The Australia Telescope is funded by the Commonwealth of Australia for operation as a National Facility managed by CSIRO.
Table 4. ATCA observation details. The array is operated in configurations with maximum baselines ranging from $75 \mathrm{~m}$ to $6 \mathrm{~km}$. For $750-\mathrm{m}$, $1.5-\mathrm{km}$ and 6-km configurations, 4 subsets (A, B, C, D) each exist.

\begin{tabular}{cllll}
\hline \hline Group & Array & Date & $\begin{array}{l}\text { Time } \\
\text { hh:mm }\end{array}$ & $\begin{array}{l}\text { Total } \\
\text { hh:mm }\end{array}$ \\
\hline SCG 2159-3210 & 750D & $1995-09-26$ & $10: 50$ & $10: 50$ \\
SCG 2353-6101 & 750D & $1995-09-23$ & $9: 52$ & $9: 52$ \\
SCG 0018-4854 & 750D & $1995-06-13$ & $4: 23$ & $17: 59$ \\
& 1.5B & $1995-05-24$ & $3: 41$ & \\
& 1.5D & $1995-10-30$ & $10: 55$ & \\
SCG 0122-3819 & 750D & $1995-09-22$ & $10: 19$ & $20: 52$ \\
& 1.5C & 2005-11-10 & $10: 33$ & \\
SCG 0141-3429 & 750D & $1995-09-25$ & $9: 58$ & $9: 58$ \\
SCG 0227-4212 & 375 & $1995-08-15$ & $10: 27$ & $10: 27$ \\
\hline
\end{tabular}

the data from the public archive and reprocessed them. In one case, SCG 0122-3819, more data were obtained in the meantime to improve the coverage of the interferometer's $u v$-plane.

All targets were observed in HI line emission with a total bandwidth of $16 \mathrm{MHz}$ subdivided into 256 channels of $62.5 \mathrm{kHz}$ and, at the same time, in $1.344 \mathrm{GHz}$ continuum with a bandwidth of $128 \mathrm{MHz}$.

The individual observing runs were normally $11-13 \mathrm{~h}$ long (including time for calibration), leading to nearly complete $12 \mathrm{~h}$ aperture syntheses. The on-source integration times for each configuration and the totals are listed in Table 4.

Simultaneously, the $1.344 \mathrm{GHz}$ continuum of the target galaxies was also imaged in the second IF (Intermediate Frequency). Because of the limited angular resolution of the resulting images, and since only integral properties were used, these images are not displayed here.

1934-638 was used as the primary flux and bandpass calibrator. The adopted flux of 1934-638 is 14.94(15.01) Jy at $1.42(1.344) \mathrm{GHz}$. The data reduction was performed in a standard fashion, using the software package MIRIAD (cf. Miriad User's Guide) and the underlying radio continuum was subtracted from the $\mathrm{H}$ I line data in the uv-plane.

HI data cubes were produced with channel widths of $125 \mathrm{kHz}$ (i.e. $26.4 \mathrm{~km} \mathrm{~s}^{-1}$ ). The H I visibilities were weighted using a robust factor of 0.5 .

\section{Results}

In this section we present the results obtained from our optical and radio pilot survey. We will first discuss the criteria used to confirm the group membership, the surrounding environment of the groups and their general properties, like mass, crossing time, and radius. Following this, we will discuss the properties of the warm medium and we will present a description of the individual targets. Within the individual targets, the properties of the member galaxies will also be discussed. 
Table 5. Classification of the compact groups and neighbouring large scale structures. The radius in Mpc is the median radius of each group, while the value given in parenthesis is the physical radius in Mpc corresponding to $15^{\prime}$. Column 4 shows the logarithm of the galaxy number density for the original galaxies in the SCGs, while in Col. 5 we show the logarithm of the galaxy number density obtained by including all galaxies within a circle of $15^{\prime}$ radius. It is worth to note the remarkable galaxy density of SCG0018-4854.

\begin{tabular}{|c|c|c|c|c|c|}
\hline Group name & Class & $\begin{array}{c}R \\
(\mathrm{Mpc}) \\
\end{array}$ & $\begin{array}{c}\text { Galaxy number density } \\
\left(\mathrm{gal} \times \mathrm{Mpc}^{-3}\right)\end{array}$ & $\begin{array}{c}\text { Galaxy number density (all galaxies) } \\
\left(\text { gal } \times \mathrm{Mpc}^{-3}\right)\end{array}$ & Notes \\
\hline SCG2159-3210 & B & $0.0436(0.081)$ & 4.06 & 3.60 & core+halo system \\
\hline SCG2353-6101 & A & 0.0841 & 3.08 & - & \\
\hline SCG0018-4854 & A & 0.0232 & 4.88 & - & \\
\hline SCG0122-3819 & B & $0.0708(0.126)$ & 3.43 & 2.77 & close to Abell $2911^{a}$ \\
\hline SCG0141-3429 & A & 0.1438 & 2.60 & - & HIPASS J0133-34 \\
\hline SCG0227-4312 & A & 0.2417 & 2.00 & - & \\
\hline
\end{tabular}

${ }^{a}$ Katgert (1996) identifies multiple redshift systems in this cluster, which at $z=0.08080$.

\subsection{Group membership and environment}

All groups in our sample have been already confirmed spectroscopically, assuming as bona fide criterion that all galaxies whose velocity difference from the median systemic velocity of the groups was less than $\pm 1000 \mathrm{~km} \mathrm{~s}^{-1}$ were group members. These galaxies are called concordant galaxies and the minimum number to confirm a group is 3 concordant galaxies. In our sample only SCG2353-6101 has three confirmed galaxies, all other targets have at least four or more concordant members.

The groups examined here have been selected, among other criteria, using an isolation criterion in the neighbourhood of the group (for more details, see Hickson 1989; and I02). We searched for neighbouring large scale structures using a $82^{\prime}$ radius, which is equal to one Abell radius at the distance of the most distant group, SCG0122-3819. In addition to this, we also add a redshift criterion: we assume that a group is close to a cluster if the velocity difference between the two is less than $3000 \mathrm{~km} \mathrm{~s}^{-1}$, i.e. one order of magnitude higher than the typical velocity dispersion of compact groups and 2.5 times higher than the largest velocity dispersion measured for clusters (1200 $\mathrm{km} \mathrm{s}^{-1}$, Zabludoff et al. 1993).

In Table 5 we show the results of our search: within our constraints, no nearby cluster has been found, but two groups are not real compact groups. SCG0122-3819 was identified by Katgert et al. (1996) as Abell 2911. Looking at their catalog however, one realizes that there are three clusters with the same name: the first, with 7 member galaxies, coincides with our group, at a redshift $z=0.02$. The second, which is in the original Abell catalog, is at redshift $z=0.08$, while a third one is at redshift $z=0.131$. Following the literature until present, we find that within an $82^{\prime}$ radius, SCG0122-3819 has 19 additional galaxies at the same redshift. Twelve of them satisfy also the requirement of having $\Delta m<3$ from the brightest group galaxy, while the other seven do not meet this requirement.

SCG2159-3210, also known as HCG90, has been studied in detail by Ribeiro et al. (1998), and defined as a core+halo system, i.e. a loose group of galaxies with a central concentration. Two of the galaxies in the loose group, $e$ and $f$, satisfy the $\Delta m<3$ criterion, while the others, $g, i$ and $o$, are fainter.

The core +halo classification for SCG2159-3210 is consistent with the extended X-ray emission of the group, well beyond the $R_{\text {group }}$ measured by Hickson. No X-ray data are available at the moment for the other groups in our sample.

We must note however, that the search radius used by de Carvalho et al. (1997) and subsequently by Ribeiro et al. (1998) is significantly smaller than our own, $15^{\prime}$ against $82^{\prime}$. Restricting the search radius to $15^{\prime}$, to be able to compare our findings with the cited work, we find that SCG0122-3819 has 7 additional galaxies, 5 of them fulfilling the $\Delta m<3$ criterion, while the other 2 are likely dwarf galaxies.

All other targets are isolated systems, confirming the goodness of the original selection. We label as class $\mathbf{A}$ all targets which can be considered really isolated on the sky, with no obvious association to larger scale structure, while we label as class B the two groups which turned out to be core+halo systems, SCG2159-3210 and SCG0122-3819.

The number density of galaxies for all our groups, as estimated in Eq. (1),

$\rho=\frac{3 N}{4 \pi R^{3}}$

is shown in Col. 4 of Table 5; in the specific cases of SCG21593210 and SCG0122-3819, the density of galaxies has been estimated both for the original galaxies in the SCGs catalog (4 for HCG90 and 5 for SCG0122-3819) and for all confirmed members within the $15^{\prime}$ radius, see Cols. 4 and 5 of Table 5 .

\subsection{Internal dynamics and mass estimates}

Having confirmed as true compact groups all our targets, with the exception of SCG2159-3210 and SCG0122-3819, we proceed to measure the characteristic properties, i.e. threedimensional velocity dispersion, crossing time, mass, luminosity and mass-to-light ratio.

For velocity dispersion and crossing time we use the same equations used in Hickson et al. (1992) and the results are shown in Cols. 3 and 5 of Table $6 . H_{\mathrm{o}} t_{\mathrm{c}}$, the dimensionless crossing time, spans from 0.017 to 0.058 , with a median value of 0.024 , which is larger than the value measured for HCGs, 0.016.

The median observed velocity dispersion is $178 \mathrm{~km} \mathrm{~s}^{-1}$, while the three-dimensional velocity dispersion is $315 \mathrm{~km} \mathrm{~s}^{-1}$, in very good agreement with what has been found for other compact group surveys, HCGs, and the DPOSS II (Pompei et al. 2006).

For the mass estimate we use different estimators, the virial and the projected mass. The expression for the virial mass is given in Eq. (2), which is valid only under the assumption of spherical symmetry.

$M_{\mathrm{V}}=\frac{3 \pi N}{2 G} \frac{\Sigma_{i} V_{z i}^{2}}{\Sigma_{i<j} 1 / R_{i j}}$

where $R_{i j}$ is the projected separation between galaxies $i$ and $j$, here assumed to be the median length of the two-dimensional galaxy-galaxy separation vector, corrected for cosmological effects. $N$ is the number of concordant galaxies in the system, and 
Table 6. Group dynamical properties. $\sigma_{r}, R, H_{\mathrm{o}} t_{\mathrm{c}}$ and $M / L$ are expressed in logarithmic values; mass and luminosity are given in solar units. All quantities for SCG0018-4854 and SCG0122-3819 have been estimated taking into account 5 member galaxies rather than four, as in the original catalog; for a more detailed explanation, see Sect. 3.5. The values in parenthesis for the $M_{\text {vir }}$ and $M_{\text {isotropic }}$ are the luminosity weighted mass estimates. The symbols follow Hickson et al. (1992).

\begin{tabular}{|c|c|c|c|c|c|c|c|c|}
\hline Group name & $\begin{array}{l}\text { Scale } \\
\left(\mathrm{kpc} /{ }^{\prime}\right)\end{array}$ & $\begin{array}{c}\sigma_{r} \\
\left(\mathrm{~km} \mathrm{~s}^{-1}\right)\end{array}$ & $\begin{array}{c}\log (R) \\
(\mathrm{kpc})\end{array}$ & $H_{\mathrm{o}} t_{\mathrm{c}}$ & $\begin{array}{c}M_{\text {vir }} \\
M_{\odot}\end{array}$ & $\begin{array}{c}M_{\text {isotropic }} \\
M_{\odot}\end{array}$ & $\begin{array}{c}L \\
L_{\odot}\end{array}$ & $M / L$ \\
\hline SCG2159-3210 & 11.34 & 1.997 & 1.640 & -1.649 & $1.89 \times 10^{12}$ & $1.02 \times 10^{12}$ & $6.20 \times 10^{10}$ & 1.22 \\
\hline SCG2353-6101 & 19.50 & 2.379 & 1.925 & -1.758 & $1.58 \times 10^{13}$ & $1.14 \times 10^{13}$ & $3.57 \times 10^{10}$ & 2.50 \\
\hline SCG0018-4854 & 14.52 & 2.065 & 1.614 & -1.735 & $3.03 \times 10^{12}$ & $1.31 \times 10^{12}$ & $4.07 \times 10^{10}$ & 1.51 \\
\hline SCG0122-3819 & 16.67 & 2.392 & 2.292 & -1.405 & $4.19 \times 10^{13}$ & $1.81 \times 10^{13}$ & $9.78 \times 10^{10}$ & 2.27 \\
\hline SCG0141-3429 & 25.92 & 2.119 & 1.994 & -1.418 & $1.43 \times 10^{13}\left(6.96 \times 10^{13}\right)$ & $6.19 \times 10^{12}\left(7.53 \times 10^{13}\right)$ & $3.22 \times 10^{10}$ & 2.37 \\
\hline SCG0227-4312 & 22.81 & 2.452 & 2.383 & -1.374 & $1.27 \times 10^{14}\left(3.98 \times 10^{13}\right)$ & $4.60 \times 10^{13}\left(4.30 \times 10^{13}\right)$ & $1.44 \times 10^{11}$ & 2.47 \\
\hline
\end{tabular}

$V_{z i}^{2}$ the velocity component along the line of sight of the galaxy $i$ with respect to the centre of mass of the group. As observed by Heisler et al. (1985) and by Perea et al. (1990), the use of the virial theorem produces the best mass estimates, provided that there are no interlopers or projection effects. In case one of these two effects is present, the current values can be considered an upper limit to the real mass.

Another good mass estimate is given by the projected mass estimator, which is defined as:

$M_{\mathrm{P}}=\frac{f_{\mathrm{P}}}{G N} \Sigma_{i} V_{z i}^{2} R_{i}$

where $R_{i}$ is the projected separation from the centroid of the system, and $f_{\mathrm{P}}$ is a numerical factor depending on the distribution of the orbits around the centre of mass of the system.

Assuming a spherically symmetric system for which the Jean's hydrostatic equilibrium applies, we can express $f_{\mathrm{P}}$ in an explicit form (Perea et al. 1990). Since we lack information about the orbit eccentricities, we estimate the mass for radial, circular and isotropic orbits and the corresponding expressions for $M_{\mathrm{P}}$ are given in Eqs. (4), (5) and (6) respectively:

$$
\begin{aligned}
& M_{\mathrm{P}}=\frac{64}{\pi G}\left\langle V_{z}^{2} R\right\rangle \\
& M_{\mathrm{P}}=\frac{64}{3 \pi G}\left\langle V_{z}^{2} R\right\rangle \\
& M_{\mathrm{P}}=\frac{64}{2 \pi G}\left\langle V_{z}^{2} R\right\rangle
\end{aligned}
$$

where $R$ is the median length of the two-dimensional galaxygalaxy separation vector.

The values for the virial mass and the projected mass estimator for isotropic orbits are shown in Cols. 6 and 7 of Table 6: the different estimators agree quite well with each other, with the exception of SCG0141-3429 and SCG0227-4312, where the virial mass is higher than the other estimators. The discrepancy would point towards the possible presence of undetected interlopers or projection effects: to test this, we re-measured all the mass estimators for these two groups, taking out one galaxy each time. For SCG0141-3429, a very good agreement between the virial mass and the other mass estimators is reached if galaxy $b$ is discarded; the same is true for SCG0227-4312 by discarding galaxy $f$. While this might lead to think that these two galaxies are likely interlopers, we must point out that the above estimators assume equal mass among all group members. Using a luminosity weighted mass calculation for both groups, and assuming $\log (M / L)=0.82$ (Roberts \& Haynes 1994 readjusted for $\left.h_{0}=0.67\right)$, we find that both virial and projected mass estimators agree very well within each other for both groups: these values are the ones quoted in parenthesis to the side of the respective unweighted masses in Table 6. The weighted values have been used for the estimate of the $M / L$ ratio in column 9 of Table 6.

The group masses vary from $1.02 \times 10^{12}$ to $4.30 \times 10^{13} M_{\odot}$, with a median value of $\sim 9.20 \times 10^{12} M_{\odot}$, very similar to the values found for Hickson's compact groups.

Group luminositites were derived by summing up the luminosity of all member galaxies. For consistency with the published works on HCGs, we estimated $B$-band luminosities: our imaging data are in $b_{j}$ (from COSMOS scans) or in $R$ (from the observations); we converted all magnitudes to standard $B$ using the equation (Prandoni et al. 1994):

$B=b_{j}+0.23 \times(B-V)$

and assuming standard $(B-V)$ colors depending on the morphological type of the member galaxies, going from $(B-V)=0.9$ for ellipticals to $(B-V)=0.5$ for irregulars. Given the closeness of the targets, morphological $k$ corrections were neglected; the derived values for the luminosity are given in Col. 8 of Table 6.

As a reference for the solar magnitude, we used the paper by Jorgensen (1994). From stars with a $(B-V) \sim 0.65$ (i.e. the same colour as the Sun) and assuming $M_{\mathrm{R}, \odot}=4.42$ (Binney \& Merrifield 2001), we obtain $M_{\mathrm{B}, \odot}=5.48$.

From the median group mass and our estimated $B$ band luminosity, we derive the mass-to-light ratio, which range from 16 to 316 , with a median value of 188 . These values are higher than for HCGs, but comparable with the values found for loose groups.

It is interesting to note that for the only group we have in common with Hickson's work (HCG90 = SCG2159-3210), we agree extremely well with his estimates for velocity dispersion, crossing time, mass and luminosity.

\subsection{Properties of the warm medium}

From the H I observations described in Sect. 2.2 we derive the following pieces of information on individual galaxies within each of the six groups tabulated in Table 1:

$$
\begin{aligned}
& \text { - H I emission distributions; } \\
& \text { - H I systemic velocities, } \\
& \text { - H I line widths, } \\
& \text { - H I total line flux densities, } \\
& \text { - H I velocity fields, } \\
& \text { - H I continuum emission. }
\end{aligned}
$$

From these data we are able to derive the H I mass, given in Eq. (8),

$M(\mathrm{HI})=2.356 \times 10^{5} D^{2} \times f(\mathrm{HI})\left[M_{\odot}\right]$,

where $D$ is the distance in Mpc. 
Table 7. Measured values of H I deficiency for SCGs in our sample. Following VM01, we assume that a group can be considered H I deficient if the difference between predicted and observed mass is more than twice the standard estimate of the error.

\begin{tabular}{lcccc}
\hline \hline Group name & $\begin{array}{c}M(\mathrm{HI})_{\text {pred }} \\
M_{\odot}\end{array}$ & $\begin{array}{c}M(\mathrm{HI})_{\text {obs }} \\
M_{\odot}\end{array}$ & s.e.e. & Def $_{\mathrm{HI}}$ \\
\hline SCG2159-3210 & $10.44 \times 10^{9}$ & $0.29 \times 10^{9}$ & 0.30 & 1.57 \\
SCG2353-6101 & $19.46 \times 10^{9}$ & $3.77 \times 10^{9}$ & 0.24 & 0.71 \\
SCG0018-4854 & $23.15 \times 10^{9}$ & $7.21 \times 10^{9}$ & 0.24 & 0.51 \\
SCG0122-3819 & $24.67 \times 10^{9}$ & $15.94 \times 10^{9}$ & 0.29 & 0.19 \\
SCG0141-3429 & $26.75 \times 10^{9}$ & $4.10 \times 10^{9}$ & 0.24 & 0.81 \\
SCG0227-4312 & $44.16 \times 10^{9}$ & $6.76 \times 10^{9}$ & 0.26 & 0.82 \\
\hline
\end{tabular}

We next derive the HI gas deficiency, by comparing with the work of Haynes \& Giovanelli (1984) on isolated galaxies.

We define the H I deficiency as:

$\operatorname{Def}(\mathrm{HI})=\log \left[M(\mathrm{HI})_{\text {pred }}\right]-\log \left[M(\mathrm{HI})_{\text {obs }}\right]$

where $M(\mathrm{HI})_{\text {pred }}$ is the predicted total H I mass for the group, obtained by summing up all the H I mass of the member galaxies, and assuming that these are field galaxies of the same Hubble type observed in the group. $M(\mathrm{HI})_{\text {obs }}$ is the observed total HI mass of the group, obtained by summing up all the H I mass measured in the member galaxies.

Values pertaining to the determination of H I deficiencies are collected in Table 7, while general H I properties of the member galaxies are tabulated in Table 8 .

We stress that the H I deficiency calculated here makes use of the H I mass, corrected by $h^{2}$, without any normalization for luminosity or diameter. In addition, we assumed that elliptical galaxies, such as SCG2159-3210B and SCG2159-3210C, have no H I gas, while we assigned to S0 galaxies their face value, as given in Table IV of Haynes \& Giovanelli (1984), without making any assumption on their possible past as spiral galaxies. The standard estimate of the error, or s.e.e., has been calculated by averaging together the individual s.e.e. for the member galaxies of each group.

Many compact groups (see VM01, for an example) are considered HI deficient, i.e. they contain less neutral gas than one would expect to find when summing up the average H I mass of field galaxies of the same Hubble type as the group members.

We find that groups in our sample have an average H I deficiency of $\operatorname{Def}(\mathrm{HI})=0.80$, see Table 7; if we exclude SCG2159-3210, which is a special case, given the presence of two elliptical galaxies and a Sy2 nucleus in one of its members, we find that the average $\mathrm{H}$ I deficiency is 0.60 , in relatively good agreement with the results by VM01.

For galaxies with SF-related activity (after exclusion of AGNs, LINERs or other types of Emission Line Galaxies [ELGs]) one can derive supernova rates, $v_{\mathrm{SN}}$ from the measured radio continuum total flux densities according to

$P_{\text {tot }}\left[\mathrm{W} \mathrm{Hz}^{-1}\right]=f_{\mathrm{SN}} \times v_{\mathrm{SN}}\left[\mathrm{yr}^{-1}\right]$.

In Eq. (10) $P_{\text {tot }}=4 \pi D^{2} f(1.34)$ is the measured total radio power derived from the radio continuum flux density, $f(1.34)$, and $f_{\mathrm{SN}}$ is a proportionality factor:

$f_{\mathrm{SN}}=\left(\frac{v}{1.5 \mathrm{GHz}}\right)^{-\frac{x}{2}}\left(\frac{B}{5 \mu \mathrm{G}}\right)^{\frac{x-2}{2}} \times 6.4 \times 10^{22}$,

in which $x$ is the radio continuum spectral index.
For a fiducial magnetic field strength of $B=5 \mu \mathrm{G}$ and a spectral index of $x=2.2$, the resulting factor is $f_{\mathrm{SN}}=7.2(2173) \times$ $10^{22}$ at the observed frequency of $1.344 \mathrm{GHz} . P_{21}^{1.34}=10^{21} P_{\text {tot }}$ is the total radio power at $1.34 \mathrm{GHz}$ in units of $10^{21} \mathrm{~W} \mathrm{~Hz}^{-1}$. This value was used for all galaxies listed in Table 9.

Based on the $v_{\mathrm{SN}}$ values one can calculate star formation rates (SFRs) for a given initial mass function (IMF). For a Salpeter IMF with an exponent of -2.35 , the following relationship exists:

$S F R=v_{\mathrm{SN}} \times \frac{2.35}{1.35} \times \frac{\left(M_{\mathrm{up}}^{-0.35}-M_{\mathrm{low}}^{-0.35}\right)}{\left(M_{\mathrm{up}}^{-1.35}-M_{\mathrm{SN}}^{-1.35}\right)}$.

For an upper and lower limit for the stellar mass range of $M_{\text {low }}=1 M_{\odot}$ and $M_{\text {up }}=100 M_{\odot}$ and a minimal mass at which a supernova can explode of $M_{\mathrm{SN}}=8 M_{\odot}$, this leads to the simple relation

$S F R=23.9 \times v_{\mathrm{SN}} M_{\odot} \mathrm{yr}^{-1}$.

Assuming a value of $S F R=0.5 M_{\odot} \mathrm{yr}^{-1}$ for quiescent galaxies (Kennicutt et al. 2005), and a threshold for a starburst: $S F R>$ $2.0 M_{\odot} \mathrm{yr}^{-1}$, we find that 6 galaxies out of 28 in our sample are star forming galaxies and 3 of these have ongoing starburst.

\subsection{Individual targets}

- SCG2159-3210 (HCG90): this is a group with four confirmed member galaxies with measured optical recession velocities, named here $a$ through $d$. Morphologies for these galaxies were obtained from Mendes de Oliveira et al. (1994) and from Hickson et al. (1989), as we lack an optical image for this group. Five additional galaxies were observed by De Carvalho et al. (1997), for a total of 9 confirmed members; however in this paper we consider only the original 4 galaxies included in Hickson's catalog and in our own.

This is a group with mixed morphology, with one spiral galaxy, $a$ (=NGC 7172), which is a Sa, two ellipticals, $b$ and $c$ and one irregular, $d$. Galaxies $a$ through $d$ have X-ray emission (Mulchaey et al. 1998); galaxy $a$ is a Sy2 (Coziol et al. 1998), while galaxies $b$ and $c$ have no optical line emission at all and galaxy $d$ is a LINER.

An additional diffuse X-ray component, with $T \sim 0.7 \mathrm{KeV}$, has been observed (Mulchaey et al. 1998; White et al. 2003), but its distribution at the moment is not very clear. In Mulchaey et al., the X-ray isophotes, which reach down to $2 \sigma$ above the background, extend along the $\mathrm{N}-\mathrm{W}$ axis of the group, almost reaching galaxy $a$, see his Fig. 1k. In the data shown by White the X-ray emission is centered on galaxies $b, c$ and $d$, with an extension towards $\mathrm{W}$.

Two of the four original members of the group, $b$ (=NGC 7176) and $d$ (=NGC 7174), are strongly interacting with each other; galaxy $d$ shows two tidal plumes, while galaxy $b$ shows a luminous bridge towards $d$ and distorted isophotes (Longo et al. 1994). White et al. (2003) demonstrate that a significant amount of optical light, $\sim 45 \%$ of the total, is in the form of a diffuse component distributed between galaxies $b, c$ and $d$.

Galaxies $a$ and $d$ have been observed in CO (Boselli et al. 1996) and the estimated mass of $\mathrm{H}_{2}$ is of the order of $10^{8.8} M_{\odot}$. The ratio between the $M\left(\mathrm{H}_{2}\right)$ and the galaxy blue luminosity is an indication of how much a galaxy has been disturbed by some kind of interaction (see again Boselli et al. 1996): we measure $\log \left(M_{\mathrm{H}_{2}} / L_{B}\right)=-1.24$ for galaxy $a$, and 


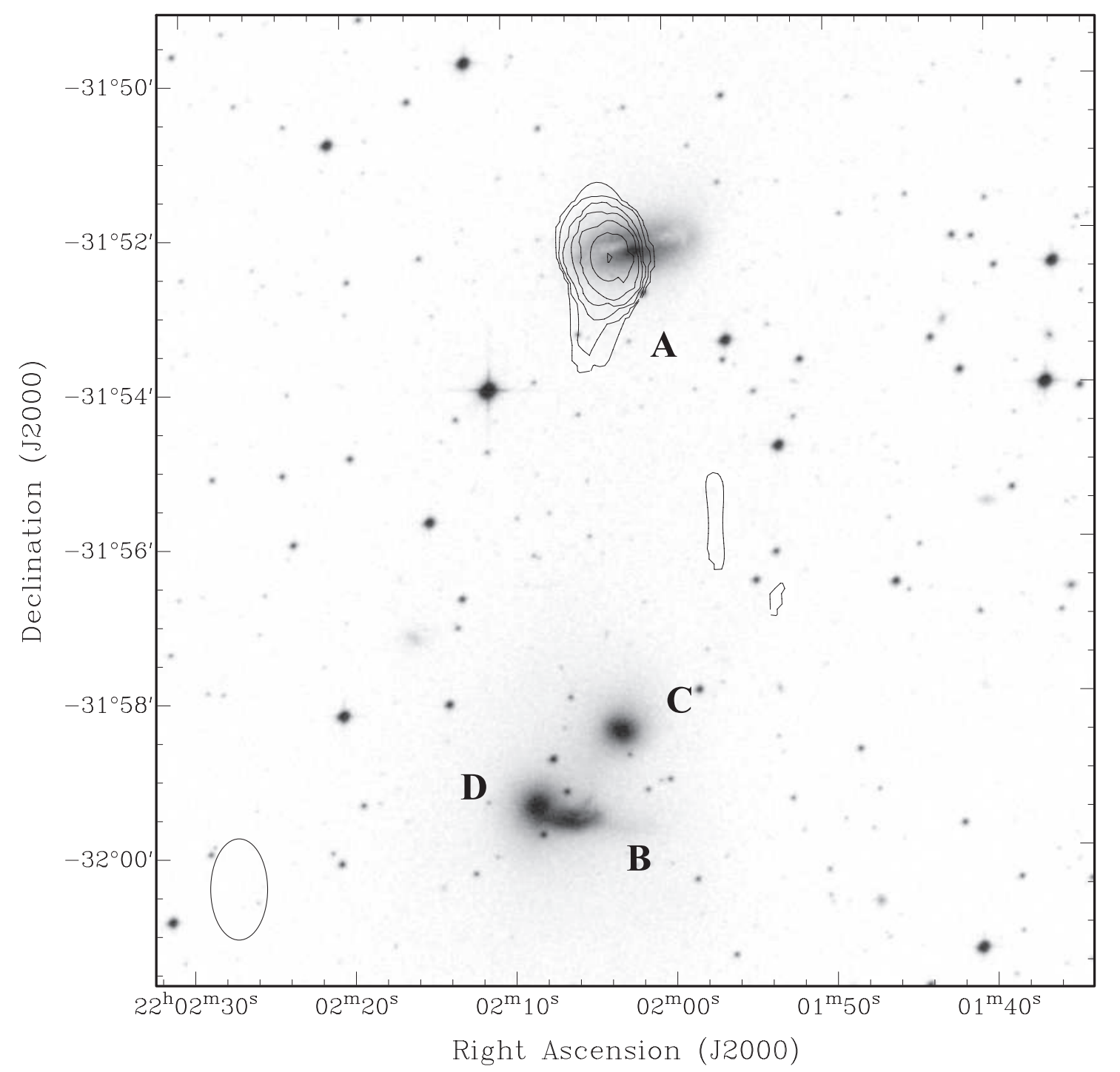

Fig. 2. ATCA H I image of SCG 2159-3210, superimposed on a red DSS-2 optical plate. The contour levels are at 0.12, 0.17, 0.24, 0.34, 0.48 0.68, and $0.96 \mathrm{Jy} \mathrm{km} \mathrm{s}^{-1}$ beam $^{-1}$. The field of view is $12.2 \times 12^{\prime} .6$ and the angular resolution $\left(F W H M=80^{\prime \prime} \times 45^{\prime \prime}\right)$ is indicated in the lower left. N is up and $\mathrm{E}$ to the left.

$\log \left(M_{\mathrm{H}_{2}} / L_{B}\right)=-0.91$ for galaxy $d$. The first value is consistent with an unperturbed galaxy, while the second is more typical of disturbed galaxies. This correlates very nicely with the morphology shown in the optical image, where galaxy $d$ is strongly interacting with $b$.

Only galaxy $a$ is detected in H I line emission, see Fig. 2: the gas distribution is highly asymmetric, with more gas in the eastern half of the galaxy disk than in the west.

Due to the presence of strong radio continuum emission from the nuclear area and beam smearing effects, no significant $\mathrm{HI}$ emission is seen in the western disk. Higher angular resolution is required to reduce this effect. Clearly the H I line flux, $f(\mathrm{HI})$, is reduced by the absorption feature, which leads to an underestimate of the total $\mathrm{H}$ I gas mass, $M(\mathrm{HI})$.

The H I emission distribution in the eastern half is disturbed, with a tidal tail protruding towards south, i.e. in the direction of the other group members. All other galaxies show no $\mathrm{HI}$ line emission at the sensitivity limit of the current data, but all of them show continuum emission: it was not possible to determine the star formation rate (SFR) from radio continuum data for galaxy $a$ because the contribution of its Sy-2 AGN cannot be assessed. Galaxy $d$ however is the strongest continuum emitter, with an estimated star formation rate of $0.92 M_{\odot} \mathrm{yr}^{-1}$ see Table 9 . It is likely that the gas reservoir which maintains the star formation comes from the molecular gas, plus cold H I gas, as picked up in absorption against the nuclear emission. An approximate estimate of the gas mass is $10^{9} M_{\odot}$, which should be enough for the central star formation activity for a limited amount of time.

The dispersion of the optical recession velocities of the four group members is only $\sim 100 \mathrm{~km} \mathrm{~s}^{-1}$, which can make tidal interactions very efficient at removing gas from galaxies.

The two-dimensional velocity field of galaxy $a$ (not displayed) shows little substructure, because most of the gas detected is located on one side of the differentially rotating part of the galaxy disk, with almost constant rotational velocities.

- SCG2353-6101: this is a small group with only 3 concordant member galaxies; galaxies $a$ and $b$ are very close to each other, and likely interacting. The optical halo of the two galaxies is touching in the middle, so masking of one of the galaxies is needed for a good photometric decomposition of the other. 
Galaxy $a$ displays two extended spiral arms, which close on each other at a radius of $\sim 46^{\prime \prime}$; a strong isophotal twist is observed. Galaxy $b$ is a diffuse spiral, with HII regions and an almost invisible bulge, while galaxy $c$ shows a boxy bulge and an exponential disk. A good photometric decomposition is obtained for all three targets using no more than two or three components, see Table 3 .

A literature search shows that both galaxies $a$ and $b$ have been observed in X-ray, but the data enable only an upper limit determination, with $L_{\mathrm{X}}<10^{40.93} \mathrm{erg} \mathrm{s}^{-1}$ and $L_{\mathrm{X}}<$ $10^{41} \mathrm{erg} \mathrm{s}^{-1}$ for galaxy $a$ and $b$ respectively (see Burstein et al. 1997).

Galaxy $a$ is a star-forming galaxy, with an optical SFR of $1.2 M_{\odot} \mathrm{yr}^{-1}$ (Crocker et al. 1996), which is also confirmed by Coziol et al. (2000). Its $\mathrm{H} \alpha$ luminosity is $L_{\mathrm{H} \alpha+[\mathrm{NII}]}=$ $10^{40.83} \mathrm{erg} \mathrm{s}^{-1}$, comparable to the X-ray upper limit. However the galaxy is only marginally detected in $\mathrm{HI}$, as can be seen in Fig. 3.

Galaxy $b$, on the other hand, has a lot more H I gas; a tidal tail is found to emanate from the western end of its disk towards north.

The extended HI distribution in galaxy $b$ and the almost overlapping optical halo between galaxies $a$ and $b$ might hint towards a past interaction between the two galaxies, which would have swept most of the gas away from the main galaxy and heated up the small remaining quantity, which is now emitting X-rays. On the other hand, the difference in recession velocity of the two galaxies for which H I measurements are available, $\sim 600 \mathrm{~km} \mathrm{~s}^{-1}$, is high (see Fig. 4).

It is not clear whether we can assume the existence of a physical gaseous bridge between $a$ and $b$; what looks like a steady velocity gradient in the data might be caused by beam-smearing, rather than a gas flow.

The last remaining galaxy in this group, which can be seen in the south-western corner of Fig. 3, does not exhibit any $\mathrm{H}$ I emission down to the sensitivity limit of the current data, see Table 8.

No good-quality radio continuum data are available due to severe confusion of the field-of-view by a nearby radio galaxy with a total flux density of $>5 \mathrm{Jy}$. Visual inspection of the resulting image shows radio continuum emission from the centre of galaxy $b$, but the data quality is too poor to further quantify this.

- SCG0018-4854: this group has four galaxies in close proximity, with a fifth member at a larger projected distance, $15^{\prime}$. All four close members show a disturbed morphology and a late type appearance: the brightest galaxy, $a$ (=NGC 92), has an extended tidal tail, with many HII regions very prominent in $\mathrm{H} \alpha$, a bright circumnuclear ring of star formation, with a pronounced knot and a strong central starburst, with a SFR, as determined from the ATCA radio continuum observations, of $14.7 M_{\odot} \mathrm{yr}^{-1}$ and a SN rate of about $0.6 \mathrm{yr}^{-1}$. The galaxy has a strong central radio continuum source, which causes the absorption hole visible in Fig. 5.

$\mathrm{HI}$ gas is clearly detected in its disk and along the whole length of its southern tidal tail.

Galaxy $b$ (=NGC 89) has strongly twisted isophotes, a Sy2 nucleus and a NE oriented jet, well visible on $\mathrm{H} \alpha$ images (Temporin, private communication). It is the only galaxy in the group not being detected in $\mathrm{HI}$ with an upper limit of $3 \times 10^{7} M_{\odot}$. However we detected radio continuum emission (Table 9), albeit fainter than galaxy $a$.

Galaxy $c(=$ NGC 87) is an irregular galaxy, with no real centre and strong star formation.
Galaxy $d$ (=NGC 88) has another active nucleus (Coziol et al. 2000); its H I emission forms a bridge towards galaxy $a$. The fifth galaxy, $e$ (=ESO198-G013), is a spiral galaxy, with diffuse arm morphology; H l line emission is detected, but the gas distribution does not show any major disturbances. Surface photometry of the galaxies has been possible only with extensive masking of nearby objects and irregular features, the results are listed in Table 3, while H I data are given in Table 8.

The relative projected velocities of the member galaxies of this group are small, resulting in a group velocity dispersion of only $120 \mathrm{~km} \mathrm{~s}^{-1}$. A large fraction of this value is contributed by galaxy $e$, which has a relative projected velocity that differs from the rest by about $200 \mathrm{~km} \mathrm{~s}^{-1}$. At the same time it is also at a large projected distance from the core of the group.

Figure 6 shows that the rotational amplitude of galaxy $a$ encompasses the total velocity range observed in both companions detected in $\mathrm{H}$ I line emission, $c$ and $d$, and the gas bridge connecting them. The NW half of galaxy $a$ is the receding side. The velocities of the HI gas in the tidal tail are an extension of those observed on that side of the galaxy disk, clearly suggesting a physical link between disk gas and tail. X-ray observations of the close members show X-ray emission mainly centered on galaxies $a$ and $b$ (Trinchieri, private communication), with a possible bridge between the two galaxies, unfortunately at a level comparable to the background. It is interesting to note an anti-correlation between $\mathrm{H}$ I and X-ray emission, with two separate bridges of hot and warm gas connecting galaxy $a$ with $b$ and $d$ respectively.

The H I velocity field of galaxy $e$ (=ESO 194-G013; not displayed) is regular. The north-eastern side of the galaxy is the receding part.

All galaxies have been observed in $\mathrm{CO}$ by Boselli et al. (1996): galaxies $a$ and $b$ contain significant amount of $M\left(\mathrm{H}_{2}\right), 10^{9.3} M_{\odot}$ and $10^{8.6} M_{\odot}$, respectively; surprisingly enough galaxy $a$ has not been detected at all at $60 \mu \mathrm{m}$ from IRAS.

The $\log \left(M_{\mathrm{H}_{2}} / L_{B}\right)$ is equal to -0.068 and -0.84 respectively for galaxies $a$ and $b$ : according to these measures, both galaxies are perturbed. Galaxies $c$ and $d$ instead have negligible amounts of molecular gas.

With two active nuclei and a strong starburst, plus two starforming galaxies, this group is the likely southern counterpart of the famous HCG 16, the youngest and most active group in Hickson's catalog, except at an higher density.

- SCG0122-3819: this group lies within $10^{\prime}$ radius from the cluster Abell 2911, which counts 31 galaxies in total (Katgert et al. 1996), but it is at a completely different redshift. Galaxies $a, b$ and $d$ are barred, while galaxy $c$ shows an extended tidal tail, very similar to that observed in SCG0018-4854a. Galaxies $a$ and $c$ are very close to each other and likely interacting: an halo of optical light between the two is clearly visible between the two objects.

A boxy bulge for galaxy $d$ was detected by Lütticke et al. (2000), but the detailed surface photometry executed with GALFIT shows that this is really an exponential bar. Actually this group is dominated by barred galaxies, $a$ and $b$, showing a flat profile (i.e. strong) bar, while $d$ has an exponential one.

Of the four optically identified member galaxies in SCG 0122-3819 only one, galaxy, $c$, is also detected in $\mathrm{HI}$ line emission (Fig. 7). This is a starburst, with a radio continuum SFR of approximately $2 M_{\odot} \mathrm{yr}^{-1}$. 
Galaxy $d$ has been observed by Pierini et al. (2003), who quote a galaxy luminosity at $1.4 \mathrm{GHz} \log \left(L_{1.4 \mathrm{Ghz}}\right)=21.64 \pm$ 0.18 , and a detection at $170 \mu \mathrm{m}$.

Our H I data prove that there is at least one more galaxy with accordant redshift, the spiral galaxy ESO 296-G 026, named galaxy $e$. This galaxy is also strongly forming stars, with a SFR of almost $2 M_{\odot} \mathrm{yr}^{-1}$.

Galaxies $c$ and $e$ are strong FIR emitters between 25 and $100 \mu \mathrm{m}$ (NED).

As already discussed in Sect. 3.1, the discovery of so many galaxies close to this group made an assesment of its true nature very difficult. We probed the entire ATCA field of view, $40^{\prime}$, for H I emission, but found no other emitter other than galaxies $c$ and $e$. The disturbed distribution of $\mathrm{H} \mathrm{I}$ emission in galaxy $e$, together with the equally disturbed distribution of galaxy $c$, led us to believe that galaxy $e$ is interacting with the group and we decided to include it in the number of member galaxies.

The estimate of the galaxy number density at different radii from the optical centre of the group shows that SCG0122-3819 is one of the low density groups and that the density decreases already by one order of magnitude by enlarging the radius to $15^{\prime}$. The strong drop in galaxy number density outside the original group circle makes this group very similar to SCG2159-3210: a central concentration of galaxies, formed by the original four targets discovered in the SCGs catalog, plus a diffuse halo of other galaxies.

Two more faint $\mathrm{HI}$ emission features are detected at $\alpha, \delta(2000)=01: 25: 22,-38: 10$ and $\alpha, \delta(2000)=01: 24: 27$, $-38: 04: 30$ respectively. These two emission features are observed at the locations of two optically faint galaxies, possibly dwarf systems. However optical spectroscopic observations put the redshift of these two galaxies at $z \sim 0.08$, confirming them as members of Abell 2911, rather than the group. We might hint that at least for one H I detection we are looking at intragroup $\mathrm{HI}$ gas not related to any specific galaxy. Table 8 lists quantities derived from the $\mathrm{HI}$ line observations.

SCG0122-3819 is another group with a relatively small velocity dispersion. The only galaxy whose velocity differs from those of the others by several hundred $\mathrm{km} \mathrm{s}^{-1}$ is $c$, which shows signs of a strong ongoing interaction. The velocity fields of both $c$ (=NGC 546), and $e$ (=ESO 296-G 026; Fig. 8) indicate that the receding side in both galaxies is the north-eastern half.

Both galaxies exhibit signs of tidal disturbances, not only in the gas distribution, but also its kinematics. Since both are viewed close to edge-on, their rotational amplitudes can be used to determine that they are normal-sized to massive spirals.

- SCG0141-3429: this group has five previously detected member galaxies, of which four were also found in H I emission. The whole group is composed of lenticulars and spirals, two of which, $b$ (=IC1722) and $c$, have strongly disturbed morphologies, with a displaced nucleus and bright knots of star formation. Residual maps in Fig. 1 clearly show the irregular distribution of bright knots in galaxy $c$, which are likely star forming regions. Both galaxies are detected by IRAS as strong infrared emitters.

Galaxies $a$ and $d$ are normal spirals, while $e$ is a late type galaxy with many SF regions. Galaxies $b$ and $c$ show strong emission lines, $\mathrm{H} \alpha$, [NII], [SII] [OIII] and [OII], while $e$ has very faint emission and off-centre with respect to the slit position, which was centered on the galaxy nucleus.
Surface photometry for galaxies $b$ and $c$ was not straightforward, due to the irregular morphology of the targets and due to the bright SF knot visible in the northern part of galaxy $c$. However good results were obtained after some trial and error and the best fitting parameters are reported in Table 3.

The HI emission properties of the four detected galaxies vary widely, see Fig. 9 and Table 8.

With the angular resolution of the current data it is not entirely clear whether the H I gas distribution in galaxy $b$ is disturbed or not. Galaxy $e$ does appear to have a tidal tail emanating from the eastern edge of its disk towards south. Although detected only with low signal-to-noise ratios, the H I gas distributions in galaxies $a$ and $c$ are clearly disturbed. Considering that galaxy $a$ is the optically brightest galaxy, it has very little H I gas left, which is completely displaced with respect to its optical body along its minor axis, towards $c$.

Radio data confirm that galaxy $c$ is actively forming stars at a rate of almost $3 M_{\odot} \mathrm{yr}^{-1}$, see Table 9 . The H I gas distribution of galaxy $c$, in turn, is highly asymmetric, with a significant fraction of the gas found in the intergalactic space south of the optical disk, roughly towards $a$. Only galaxy $d$, although confirmed from optical spectroscopy to have a recession velocity similar to the other group members, is not detected in $\mathrm{HI}$ at all. Note that, although listed as an IRAS far-infrared source, galaxy $d$ is not detected in $1.34 \mathrm{GHz}$ radio continuum emission either (Table 9).

Quantitative HI measurements for all member galaxies are listed in Table 8.

The velocity dispersion of the member galaxies of SCG 0141-3429 is small, with four galaxies involved in tidal interactions at the same time, which makes it similar to the Grus quartet of galaxies (SCG2315-4241, cf. Dahlem 2005). Despite the strongly disturbed H I gas distributions, the velocity fields (not displayed here) are still quite regular, indicating ordered, large-scale gas flows, as it is shown in Fig. 10.

- SCG0227-4312: all group members are spiral galaxies and, with a total of six optically identified concordant members, SCG 0227-4312 is the richest compact group presented here. The member galaxies show no signs of optical disturbances, but three galaxies, $c, d$ and $f$ show either a bar or a lens. Galaxy $c$ is the only one which also show prominent spiral arms which close around the external ring at the end of the bar at $\sim 33.5^{\prime \prime}$, see Table 3 . All six members of SCG 0227-4312 can be seen in Fig. 11.

Of these, only three are detected in $\mathrm{H}$ I line emission, namely $b, c$ and $d$. With the angular resolution of the data presented here no large-scale irregularities are visible in the HI gas distributions of these three galaxies, while all show signs of small disturbances. The early-type galaxy $a$ shows no H I line emission at all and, more surprisingly, neither do the edge-on spirals $e$ and $f$, see Table 8 for quantitative results.

Only one galaxy, $e$, which is classified as a star forming galaxy, has a detectable radio continuum flux density, indicating a moderate SFR of around $1 M_{\odot} \mathrm{yr}^{-1}$, while all others are very faint at $1.34 \mathrm{GHz}$.

Both the projected distances and the velocity dispersion of SCG 0227-4312 (281 $\left.\mathrm{km} \mathrm{s}^{-1}\right)$ are large compared to other groups presented here, see Fig. 12 and Table 8.

This may explain why this group shows no direct signs of ongoing interactions. The velocity fields of the three galaxies showing $\mathrm{H}$ I emission are roughly regular. Note that, despite the wide spread in total H I gas masses, the rotational amplitudes of the three galaxies detected in $\mathrm{H} \mathrm{I}$ emission are 
quite similar (Table 8). For similar inclinations, this would indicate comparable total masses.

\section{Discussion}

With the information coming from our optical and radio pilot sample, we can now ask the following questions:

- how does the population of our SCGs compare with HCGs?

- do we see any special feature in the intragroup medium?

- what can we say about group evolution?

Before answering the first question, we must point out that, being a pilot study, the number of groups presented here is not representative of the complete SCGs catalog and definitely not enough for any statistical conclusion. It is interesting however to have an idea of what kind of galaxies we are looking at with respect to the average properties of compact group galaxies.

In terms of morphological classification, we have that 16 galaxies out of 28 , i.e. $60 \%$, are $\mathrm{Sa}$ or later, while the others are either E or S0. Nine out of 28 galaxies in the groups are highly irregular, with signs of strong morphological disturbances, which corresponds to $\sim 30 \%$ of the total number of galaxies. Eleven galaxies show strong bars, which could be fitted with a Sersic profile, often with a pronounced external ring.

AGN activity, either in the form of a LINER or an AGN, has been found in seven galaxies, i.e. about $\sim 25 \%$ of our sample, in reasonable agreement with Coziol et al. (2004); we should point out that the classification of different types of nuclear activity, as entered into the Remarks column of Table 3, was revised compared to those published by Coziol et al. (2000). For the revised classification of galaxies in SCG 0018-4854 see Tanvuia (2005), while the classification for SCG0141-3429 is presented here for the first time.

About 10 galaxies, $\sim 35 \%$, show measurable star formation in the optical, but only one, SCG0018-4854A, is a starburst. On the other hand, using radio continuum data, and assuming as a threshold for starburst a SFR of $2 M_{\odot} \mathrm{yr}^{-1}$, we find that 6 galaxies out of 28 can be defined star forming galaxies, and half of these six are starbursts. The difference between the number of star-forming and starburst galaxies identified in the optical vs. radio regime arises from the fact that we determine SFRs in different ways. Optical SFRs come from long-slit spectra probing only the central regions of the galaxies, in order to determine the dominant type of nuclear activity, if any. On the other hand, radio SFRs were calculated based on integral radio continuum flux densities summed up over the surface areas of entire galaxies. These are therefore global SFRs, rather than measures of nuclear activity.

From these results, we can preliminarily conclude that the SCGs presented here are on average richer in spiral members than HCGs; however more data should be taken into consideration before considering this a firm conclusion. SCGs show an higher level of central star formation, but are comparable with HCGs in terms of occurrence of nuclear activity (i.e. LINER and AGNs). We suggest that this confirms the hypothesis that SCGs are on average younger than HCGs. This is likely to be due both to the different surveys from which the two samples were selected and to the different selection criteria used for the SCGs and HCGs. Hickson's groups were selected on red plates, while the SCGs catalog has been defined through the COSMOS survey, i.e. blue plates, likely to highlight younger galaxies. In addition to this, the automatic group identification used to find SCGs, opposed to the eyeball search for HCGs, has eliminated the bias of selecting preferentially higher surface brightness groups and groups with two or more galaxies of comparable magnitude, which is present in the HCGs (Prandoni et al. 1994).

The sensitivity of $\mathrm{HI}$ observations is about $2 \times 10^{8} M_{\odot}$; however twelve (43\%) of the optically identified 28 member galaxies in the six groups are not detected in HI line emission. Of these 12, 8 galaxies are classified as early-type (E or S0; SCG2159-3210b and $c$, SCG2353-6101c, SCG0122-3819b and $d$, SCG0141-3429 $d$ and SCG0227-4312 $a$ and $e$ ).

On the other hand, 4 galaxies without $\mathrm{H}$ I detections are later types in which one would normally expect to find atomic hydrogen gas. While for SCG2159-3210d and SCG0122-3819a this can be explained by stripping caused by the strong interaction these galaxies are experiencing, it is very difficult to understand what has happened to galaxy SCG0227-4312f, which seems completely undisturbed and should not have such $\mathrm{H}$ I deficiency. A search of the Chandra and XMM archives returned no result on this galaxy, so at the moment we are left with an unsolved riddle.

This lack of gas is confirmed by the fact that we find an average HI deficiency of 0.61 . We would like to stress that this deficiency is an upper limit to the real estimate of H I deficiency in groups, as we did not apply any correction for H I absorption for the presence of an active nucleus or thick gas.

Tidal tails are more evident in H I observations than in the optical, with 9 galaxies showing such feature in the H I gas distributions, while only three have optical tidal tails. Other signs of morphological disturbances can be detected in another 4 galaxies, bringing the total of interacting galaxies to 13 , almost half of the sample. From these results, we can conclude that $\mathrm{HI}$ is a very powerful tool to trace the effects of the interactions among the member galaxies out to a large distance from the centre of the galaxies themselves.

H I data give more information on the dynamical evolutionary state of the groups: following the classification scheme by VM01, we can identify which phase the six groups presented here are currently in, from the so called phase 1, with the gas mainly centered on the member galaxies, to phase 2, with extended tidal tails and perturbed gas distribution to phase $3 a$, with almost no or very little $\mathrm{H}_{\mathrm{I}}$ left, or phase $3 b$, with all the $\mathrm{H}$ I gas distributed in the group potential well and not centered on any single galaxy.

SCG 2159-3210 is at a late stage of phase $3 \mathrm{a}$ in its dynamical evolution. No H I is found in the three strongly interacting galaxies in this group and the H I gas in galaxy $a$ exhibits an asymmetric distribution and a tidal tail. This group is extremely deficient in H I gas, because almost none was detected in its member galaxies. The evolutionary stage finds further confirmation in the group-wide X-ray emission (Ponman et al. 1996).

SCG 2353-6101, on the other hand, must still be in an early stage of its dynamical evolution, because there are only few indications that the H I gas in its galaxies is disturbed.

The four galaxies near the centre of SCG 0018-4854 have a common H I envelope, which indicates that they are in phase $3 \mathrm{~b}$ according to VM01. Galaxy $e$, farther away from the central four, is not part of the ongoing interactions and it is located outside their common envelope.

Although both SCG 0122-3819c and SCG 0122-3819e show signs of interactions in the form of tidal tails, these are not very pronounced yet and their H I gas is still well aligned with their stellar disks. Therefore, this group is most likely still in phase 1 of its dynamical evolution, possibly just starting to move into phase 2 . 
SCG 0141-3429 is far more developed than SCG 0122-3819. The two galaxies $a$ and $c$ have lost, or are in the process of losing, most of their H I gas into the intergalactic space, with asymmetric $\mathrm{HI}$ distribution and tidal tails. This would place this group in phase 2, having galaxies $b$ and $e$ at an earlier stage of their development, with $\mathrm{H}$ I that is slightly disturbed, but still tightly bound to the stellar disks.

SCG 0227-4312 is still dynamically young, in phase 1 of its evolution. The late-type galaxies show little, if any, sign of gravitational disturbance in their $\mathrm{H}$ I distributions and kinematics. The only galaxies that have no H I are early-type galaxies, with the exception of galaxy $f$. According to Casasola et al. (2004) this is further evidence that these galaxies did not experience any interaction at all.

From the above it is clear that a classification of each group into a specific evolutionary stage is not easy. Nevertheless, the data show that some groups are still in early stages of their evolution, while others are already quite evolved. It should be noted that the group in phase $3 \mathrm{~b}$ does not show H I deficiency; the same is true for the similarly classified groups in VM01, see for example HCG49. Another interesting point is that both Sy2 in our sample belong to groups in the last evolutionary phase. However, while SCG2159-3210 $a$ is located outside the main site of interaction and it has retained some of its H I gas, SCG0018-4854b is sitting in the middle of an interacting group and it is completely devoid of H I. The different H I gas distributions observed in the SCGs presented here highlight the importance of $\mathrm{H}$ I gas as diagnostic for studies of the dynamical evolution of galaxy groups.

\section{Conclusions}

In this pilot study of six compact groups drawn from the Southern Compact Groups catalog we have found that;

- Four groups are real compact groups, while two are core+halo systems.

- The three dimensional velocity dispersion and mass of our groups are very similar to HCGs; the range covered in crossing time and $\mathrm{M} / \mathrm{L}$ is compatible with the distribution observed for HCGs.

- Out of 28 galaxes, $35 \%$ of them are forming stars in their central region, and approximately $60 \%$ of them are spirals of morphological type Sa or later.

- Six galaxies, about $20 \%$ of the total, are forming stars throughout the whole galaxy and three of them are starbursts.

- Half of the galaxies show signs of interactions.

- The HI distribution in our groups approximately reveal the presence of all the four evolutionary stages highlighted by VM01. The average H I deficiency is in agreement with what has been measured for galaxes in VM01.

More detailed studies of individual groups will be conducted by us, based on multi-wavelength observations. At the same time, we will be collecting a more complete database of all groups in our statistically complete sample comprising a total of 50 SCGs, whose brightest galaxy has $b_{j} \leq 14.5$, to increase the statistical significance of our results.

The sample of SCGs presented here is too small to perform detailed comparisons with other, larger samples. General trends found in larger samples, such as enhanced levels of nuclear activity in interacting galaxies and $\mathrm{H}$ I deficiencies in groups at late stages of their dynamical evolution, are tentatively corroborated. However, firmer conclusions will have to await the availability of a larger number of suitable data sets. As part of this, more H I observations of SCGs will be carried out and presented at a later stage.

Acknowledgements. M.D. gratefully acknowledges the kind hospitality and travel grant received from ESO during a visit to Santiago de Chile, where part of the work presented here was carried out. The Digitized Sky Survey was produced at the Space Telescope Science Institute under U.S. Government grant NAG W-2166. The National Geographic Society - Palomar Observatory Sky Atlas (POSS-I) was made by the California Institute of Technology with grants from the National Geographic Society.

\section{References}

Binney, J., \& Merrifield, M. 2001, Galactic astronomy (Princeton University Press)

Boselli, A., Mendes de Oliveira, C., Balkowski, C., Cayatte, V., \& Casoli, F. 1996, A\&A, 314, 738

Burnstein, D., Jones, C., Forman, Marston, A. P., \& Marzke, R. O. 1997, ApJS, 111,163

Casasola, V., Bettoni, D., \& Galletta, G. 2004, A\&A, 422, 941

Coziol, R., Iovino, A., \& de Carvalho, R. R. 2000, AJ, 120, 47

Coziol, R., Brinks, E., \& Bravo-Alfaro, H. 2004, AJ, 128, 68

Coziol, R., De Carvalho, R. R., Capelato, H.V., \& Ribeiro, A. L. B. 1998, ApJ, 506,545

Crocker, D. A., Baugus, P. D., \& Buta, R. 1996, ApJS, 105, 353

Dahlem, M. 2005, A\&A, 429, L5

De Carvalho, R. R., Ribeiro, A. L. B., Capelato, H. V., \& Zepf, S. 1997, ApJS, 110,1

Hamuy, M., Walker, A. R., Suntzeff, N. B., et al. 1992, PASP, 104, 533

Hamuy, M., Suntzeff, N. B., Heathcote, J. R., et al. 1994, PASP, 106, 566

Haynes, M., \& Giovanelli, R. 1984, AJ, 89, 758

Heisler, J., Tremaine, S., \& Bahcall, J. 1985, ApJ, 298, 8

Hickson, P. 1982, ApJ, 255, 382

Hickson, P., Kindl, E., \& Auman, J. R. 1989, ApJS, 70, 687

Hickson, P., Mendes de Oliveira, C., Huchra, J. P., \& Palumbo, G. G. 1992, ApJ, 399,353

Hummel, E., van der Hulst, J. M., Kennicutt, R. C., \& Keel, W. C. 1990, A\&A, 236,333

Iovino, A. 2002, AJ, 124, 2471

Jorgensen, I. 1994, PASP, 106, 967

Katgert, P., Mazure, A., Perea, J., et al. 1996, A\&A, 310, 8

Kennicutt, R. C., Lee, J. C., Funes, J. G., Sakai, S., \& Akiyama, S. 2005, Ap\&SS, 329,187

Longo, G., Busarello, G., Lorenz, H., Richter, G., \& Zaggia, S. 1994, A\&A, 282, 418

Luetticke, R., Dettmar, R. J., \& Pohlen, M. 2000, A\&AS, 145, 405

Maia, M. A. G., Pastoriza, M. G., Bica, E., \& Dottori, H. 1994, ApJS, 93, 425

Mathewson, D. S., Ford, V. L., \& Buchhorn, M. 1992, ApJS, 81, 413

Mendes de Oliveira, C., \& Hickson, P. 1994, ApJ, 427, 684

Mulchaey, J. S., \& Zabludoff, A. I. 1998, ApJ, 496, 73

Oosterloo, T., \& Iovino, A. 1997, PASA, 14, 48

Peng, C. Y., Ho, L., Impey, C. D., \& Rix, H. W. 2002, AJ, 124, 266

Perea, J., del Olmo, A., \& Moles, M. 1990, A\&A, 237, 319

Pierini, D., Popescu, C. C., Tuffs, R. J., \& Volk, H. J. 2003, A\&A, 409, 907

Pompei, E., Iovino, A., \& de Carvalho, R. R. 2006, A\&A, 445, 857

Ponman, T. J., Bourner, P. D. J., Ebeling, H., \& Bohringer, H. 1996, MNRAS, 283,690

Prandoni, I., Iovino, A., \& MacGillivray, H. T. 1994, AJ, 107, 1235

Ribeiro, A. L. B., De Carvalho, R., Capelato, H. V., \& Zepf, S. E. 1998, ApJ, 497, 72

Roberts, M. S., \& Haynes, M. P. 1994, A\&ARA, 32, 115

Tanvuia, L. 2005, Ph.D. Thesis, University of Vienna, Austria

Temporin, S., Ciroi, S., Iovino, A., et al. 2005, Ap\&SS, 329, 78

Verdes-Mentenegro, L., Yun, M. S., Williams, B. A., et al. 2001, A\&A, 377, 812

White, P. M., Bothun, G., Guerrero, M., West, M. J., \& Barkhouse, W. A. 2003, ApJ, 585, 739

Zabludoff, A. I., Geller, M., Huchra, J., \& Vogeley, M. 1993, AJ, 106, 1273 
E. Pompei et al.: Optical and radio study of six southern compact groups, Online Material p 1

\section{Online Material}


E. Pompei et al.: Optical and radio study of six southern compact groups, Online Material p 2

The next nine figures show the optical images and the GALFIT residual images for the member galaxies of the groups presented here. The following 10 additional figures show the results obtained from the radio data, overlapped on the optical images.
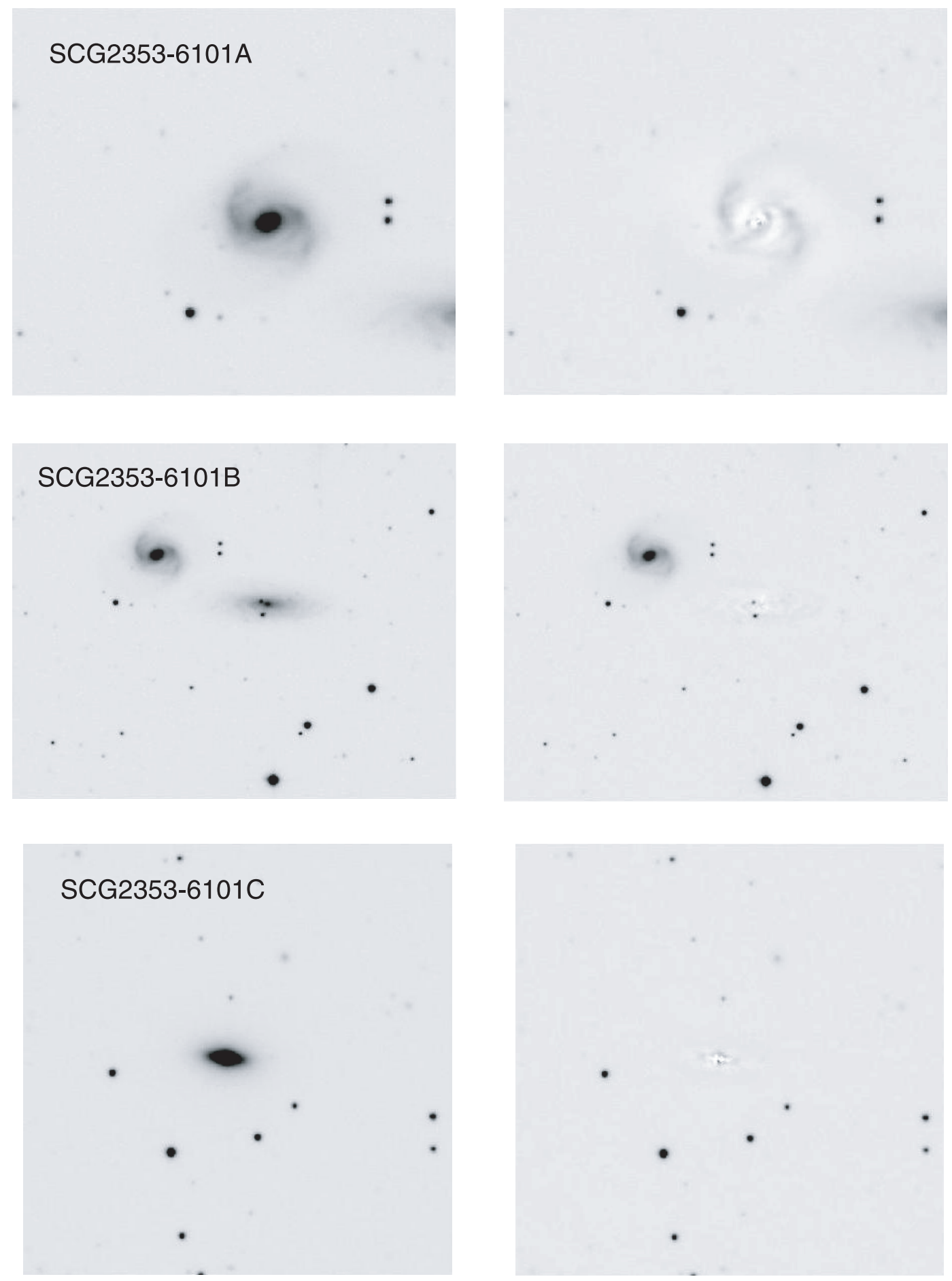

Fig. 1. Optical images (left panel) and GALFIT residual images (right panel) for SCG2353-6101, from galaxy $a$ to $c$ from top to bottom. Residual brightness for galaxy $\mathrm{A}$ is of the order of $5 \%$ of the peak brightness, while for the other galaxies is less than $1 \%$. In all images $\mathrm{N}$ is up and $\mathrm{E}$ is left. 
E. Pompei et al.: Optical and radio study of six southern compact groups, Online Material $p 3$
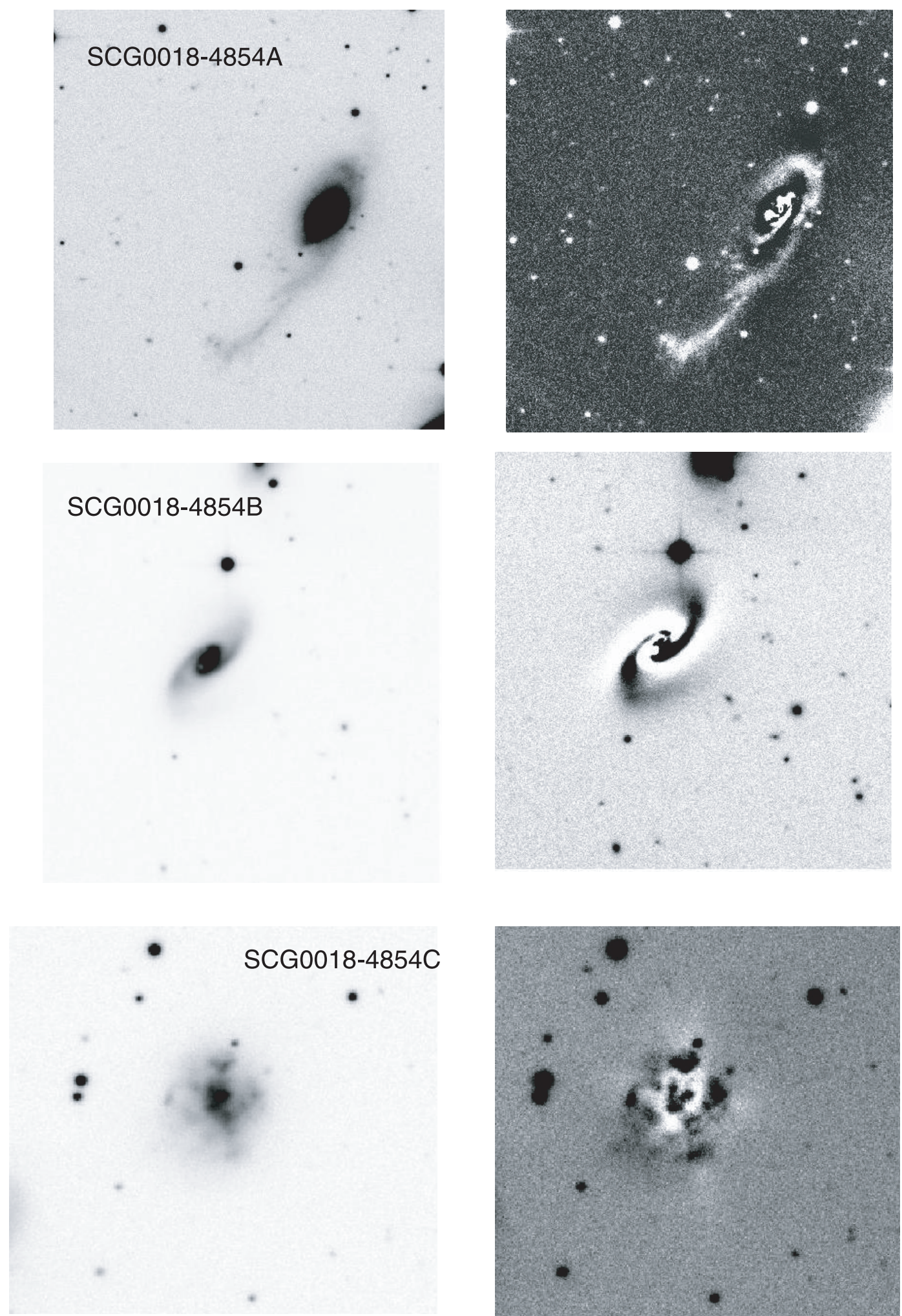

Fig. 1. continued. Optical images (left panel) and GALFIT residual images (right panel) for SCG0018-4854, from galaxy $a$ to $c$ from top to bottom. Residual brightness for all galaxies is of the order of $10 \%$ of the peak brightness. In all images $\mathrm{N}$ is up and $\mathrm{E}$ is left. 
E. Pompei et al.: Optical and radio study of six southern compact groups, Online Material p 4
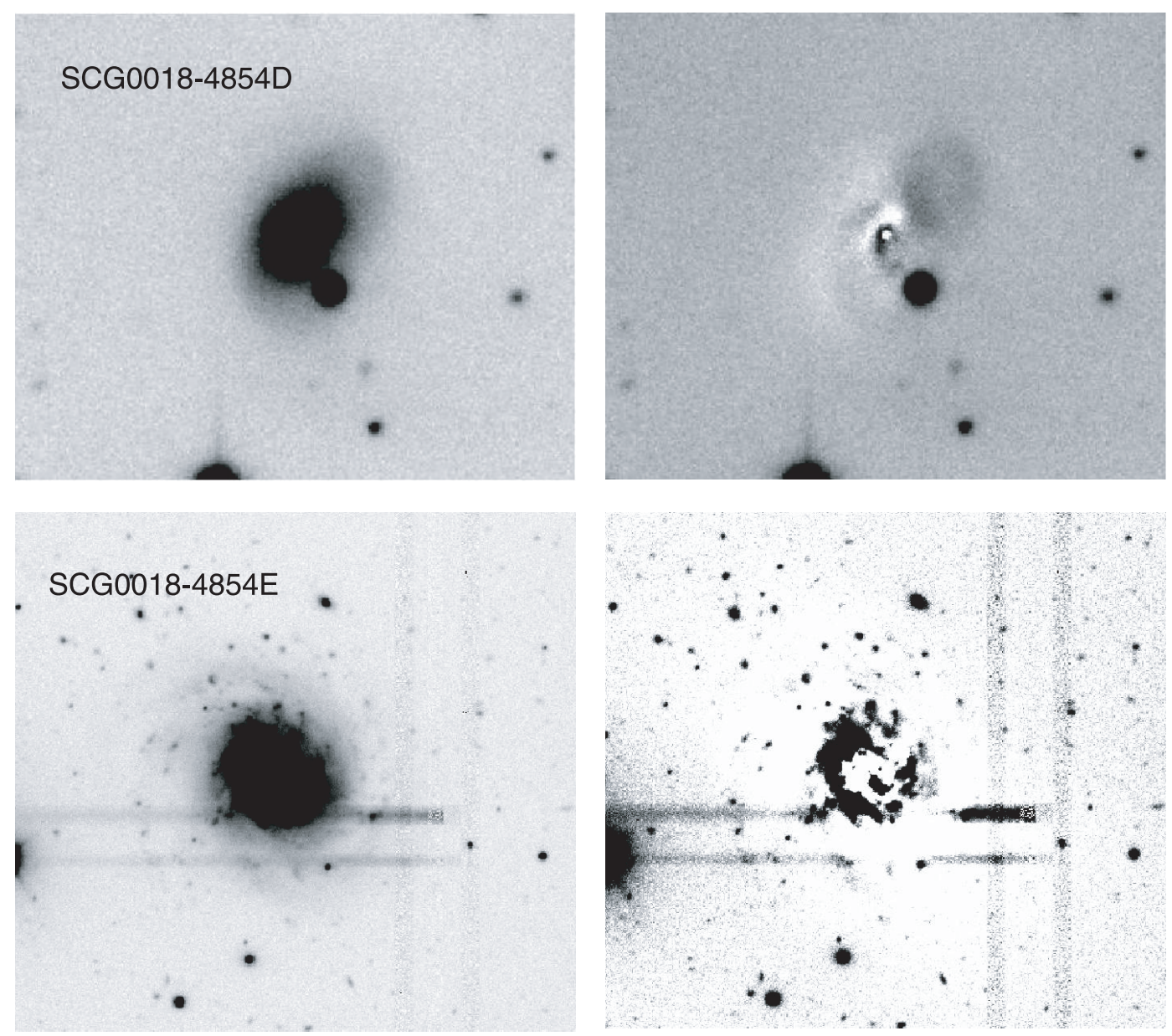

Fig. 1. continued. Optical images (left panel) and GALFIT residual images (right panel) for SCG0018-4854, from galaxy $d$ to $e$ from top to bottom. Residual brightness for all galaxies is of the order of $7 \%$ of the peak brightness. In all images $\mathrm{N}$ is up and $\mathrm{E}$ is left. 
E. Pompei et al.: Optical and radio study of six southern compact groups, Online Material p 5
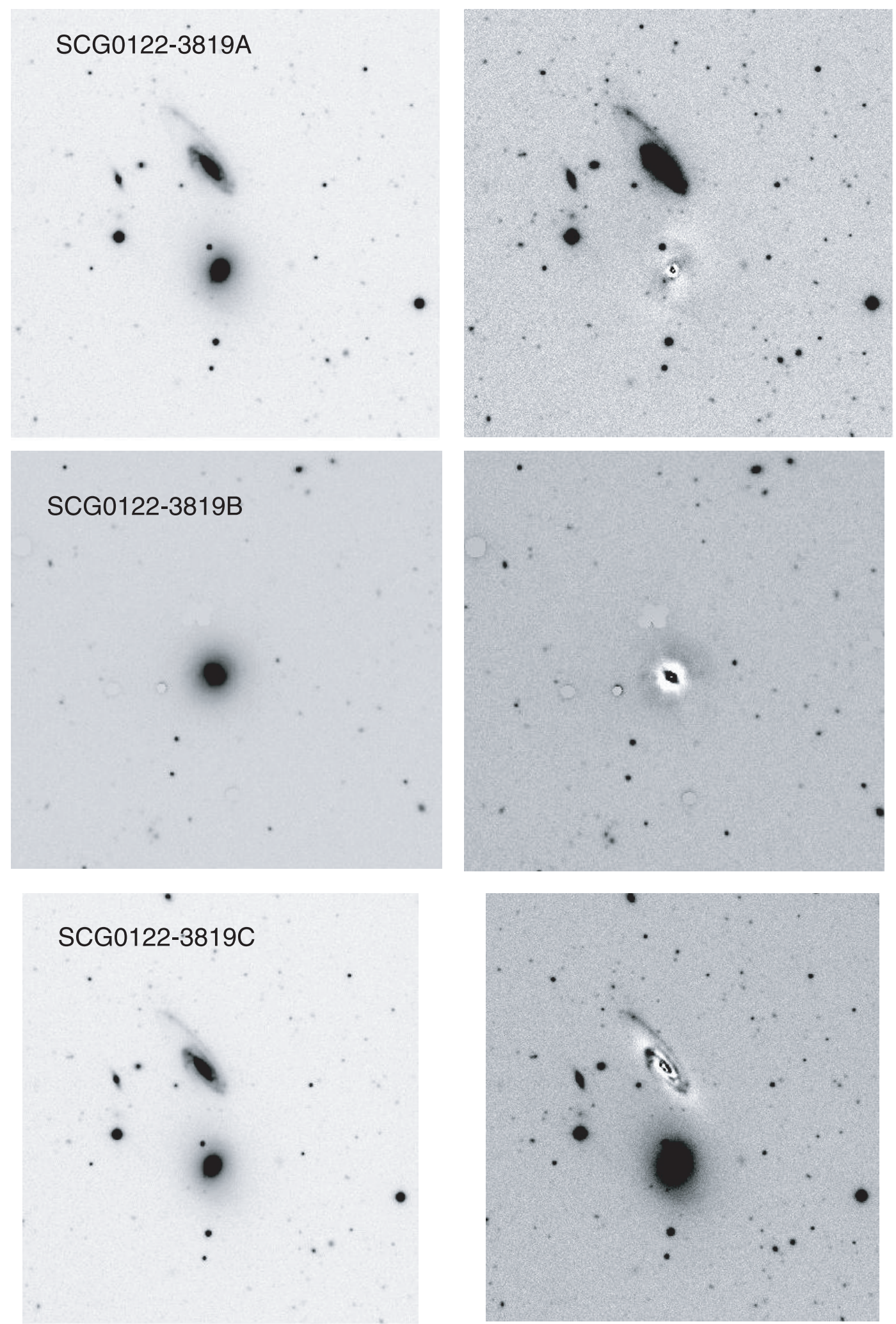

Fig. 1. continued. Optical images (left panel) and GALFIT residual images (right panel) for SCG0122-3819, from galaxy $a$ to $c$ from top to bottom. Residual brightness for all galaxies is of the order of $5 \%$ of the peak brightness, with the exception of galaxy $\mathrm{B}$, where the residuals are of the order of $15 \%$. In all images $\mathrm{N}$ is up and $\mathrm{E}$ is left. 
E. Pompei et al.: Optical and radio study of six southern compact groups, Online Material p 6
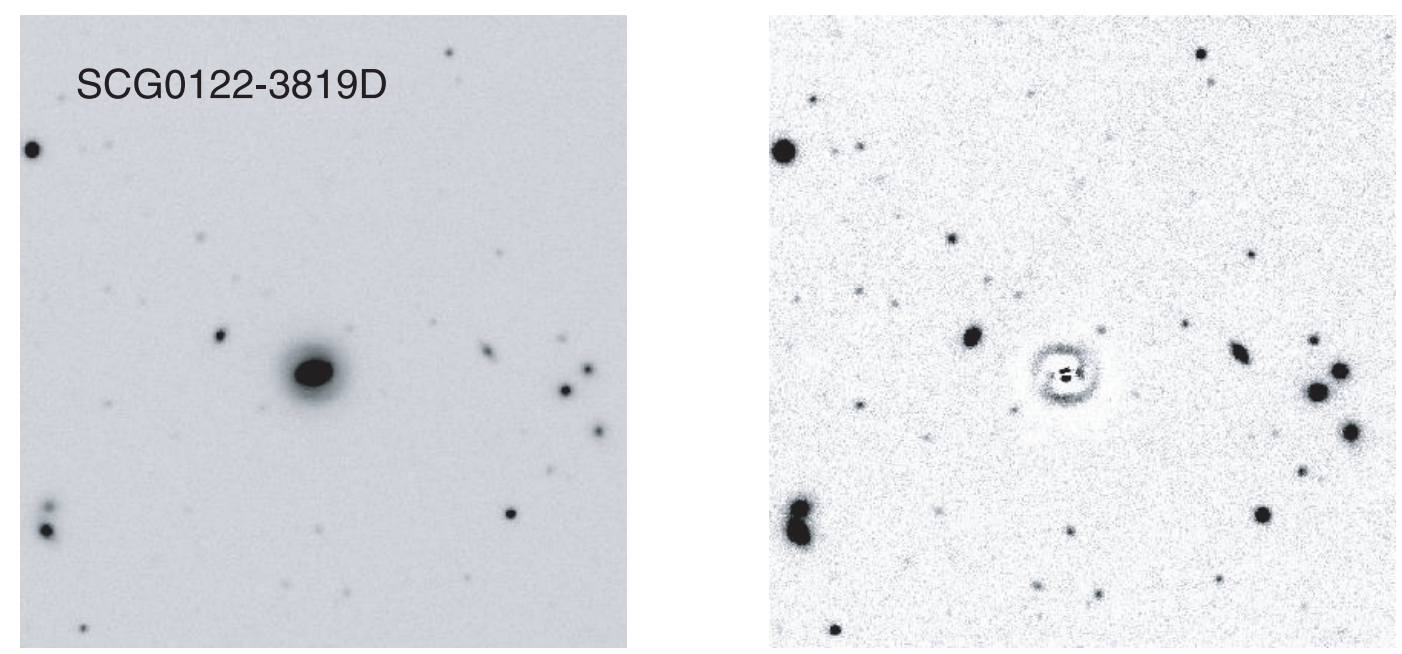

Fig. 1. continued. Optical images (left panel) and GALFIT residual images (right panel) for SCG0122-3819d; residuals are of the order of $8 \%$ of the peak brightness. $\mathrm{N}$ is up and $\mathrm{E}$ is left. 
E. Pompei et al.: Optical and radio study of six southern compact groups, Online Material $p 7$
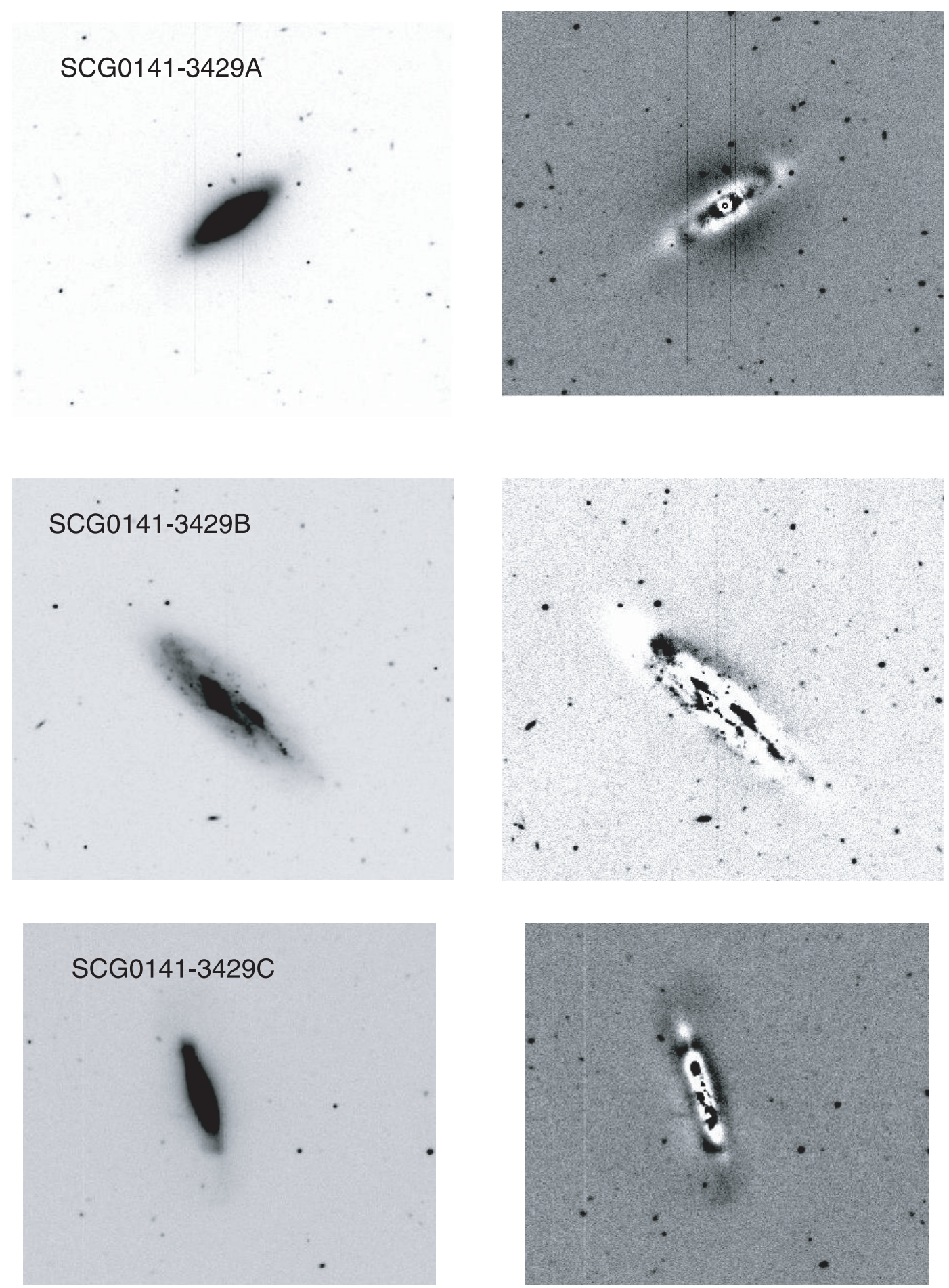

Fig. 1. continued. Optical images (left panel) and GALFIT residual images (right panel) for SCG0141-3429, from galaxy $a$ to $c$ from top to bottom. Residual brightness for all galaxies is of the order of $12 \%$ of the peak brightness. In all images $\mathrm{N}$ is up and $\mathrm{E}$ is left. 
E. Pompei et al.: Optical and radio study of six southern compact groups, Online Material $p 8$
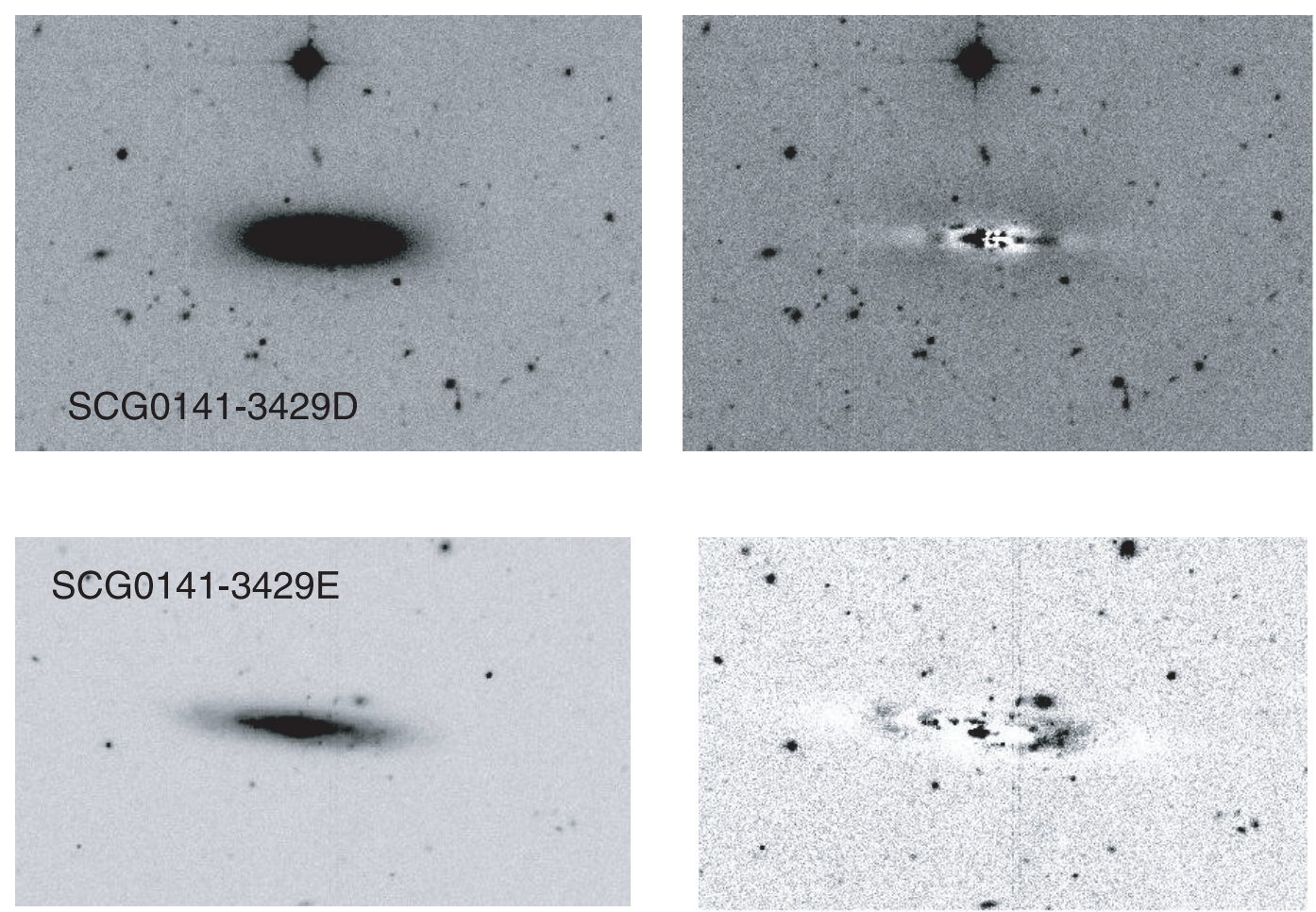

Fig. 1. continued. Optical images (left panel) and GALFIT residual images (right panel) for SCG0141-3429, from galaxy $d$ to $e$ from top to bottom. Residual brightness for all galaxies is of the order of $10 \%$ of the peak brightness. In all images $\mathrm{N}$ is up and $\mathrm{E}$ is left. 
E. Pompei et al.: Optical and radio study of six southern compact groups, Online Material $p 9$
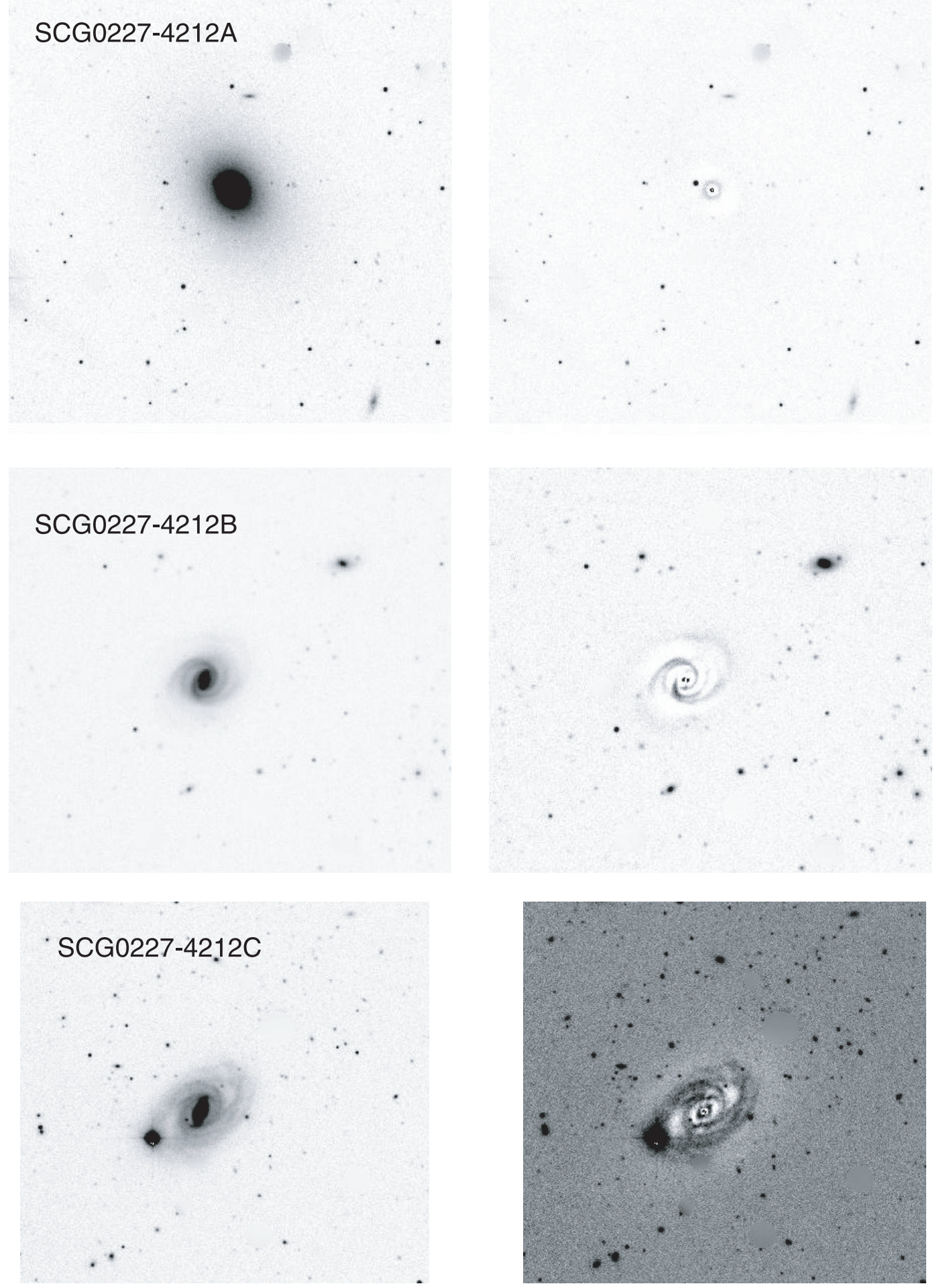

Fig. 1. continued. Optical images (left panel) and GALFIT residual images (right panel) for SCG0227-4312, from galaxy $a$ to $c$ from top to bottom. Residual brightness for all galaxies is of the order of $3 \%$ of the peak brightness, with the exception of galaxy $\mathrm{C}$, where the residuals are of the order of $8 \%$. In all images $\mathrm{N}$ is up and $\mathrm{E}$ is left. 
E. Pompei et al.: Optical and radio study of six southern compact groups, Online Material $p 10$
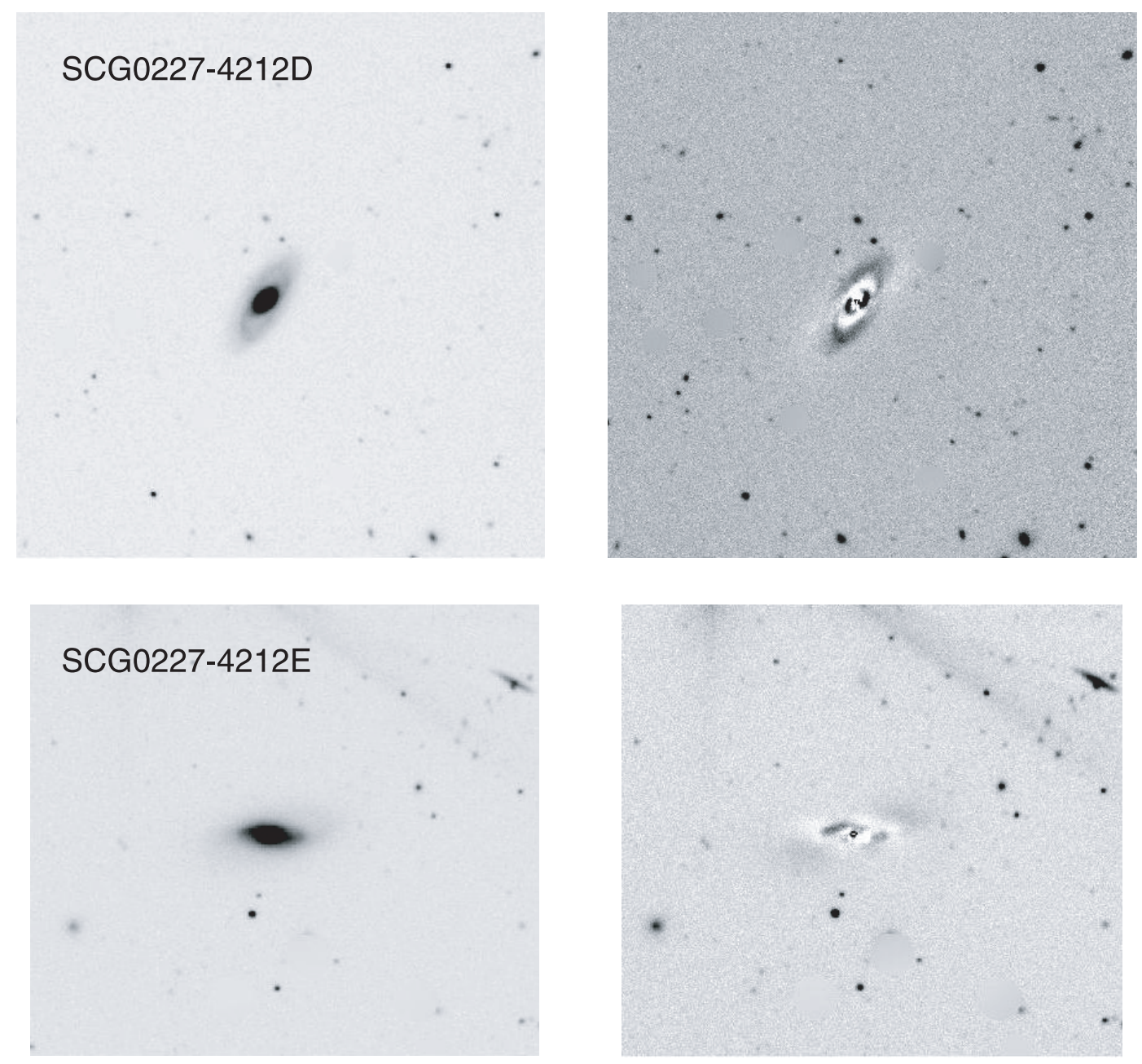

\section{SCG0227-4212F}

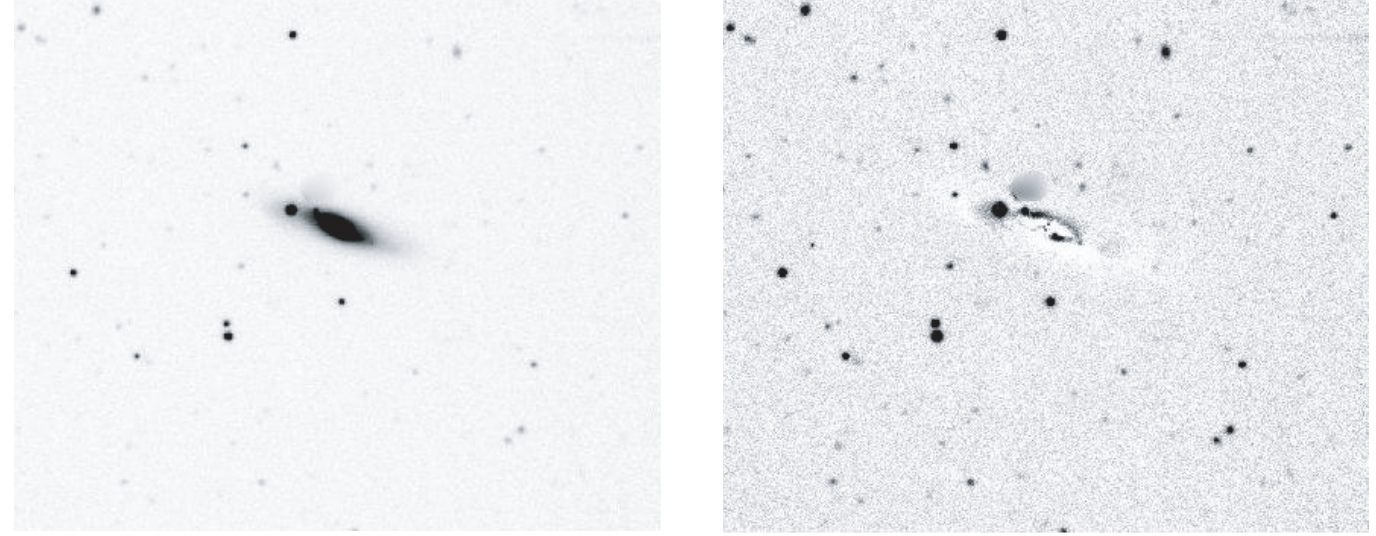

Fig. 1. continued. Optical images (left panel) and GALFIT residual images (right panel) for SCG0227-4312, from galaxy $d$ to $f$ from top to bottom. Residual brightness for all galaxies is of the order of $3 \%$ of the peak brightness. In all images $\mathrm{N}$ is up and $\mathrm{E}$ is left. 
E. Pompei et al.: Optical and radio study of six southern compact groups, Online Material $p 11$

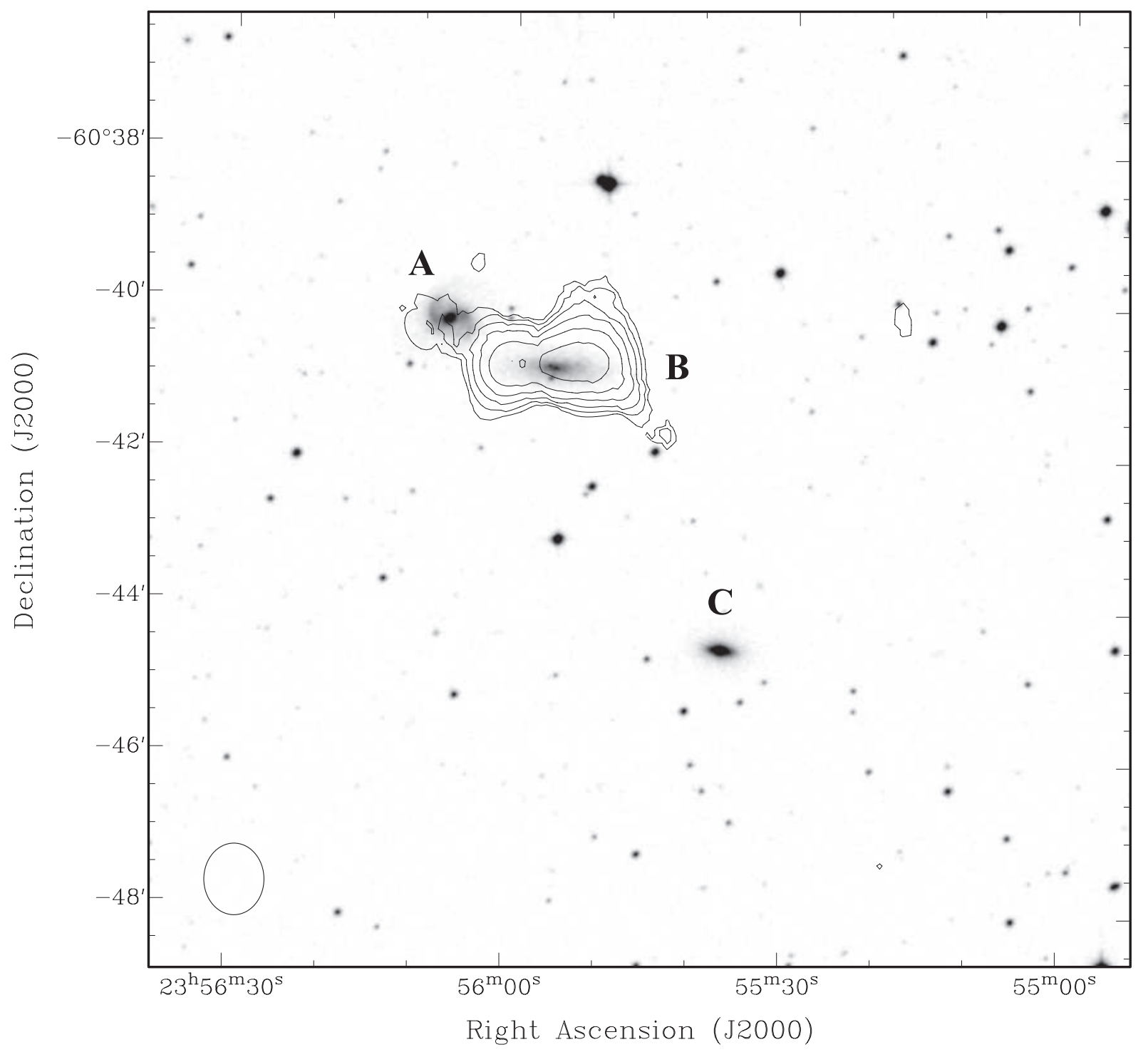

Fig. 3. ATCA H I image of SCG 2353-6101, superimposed on a red DSS-2 optical plate. The contour levels are at 0.24, 0.34, 0.48, 0.68, 0.96 and $1.36 \mathrm{Jy} \mathrm{km} \mathrm{s}^{-1}$ beam $^{-1}$. The field of view is $13.0 \times 12^{\prime} .6$ and the angular resolution $\left(F W H M=50^{\prime \prime} \times 42^{\prime \prime}\right)$ is indicated in the lower left. $\mathrm{N}$ is up and E to the left. 


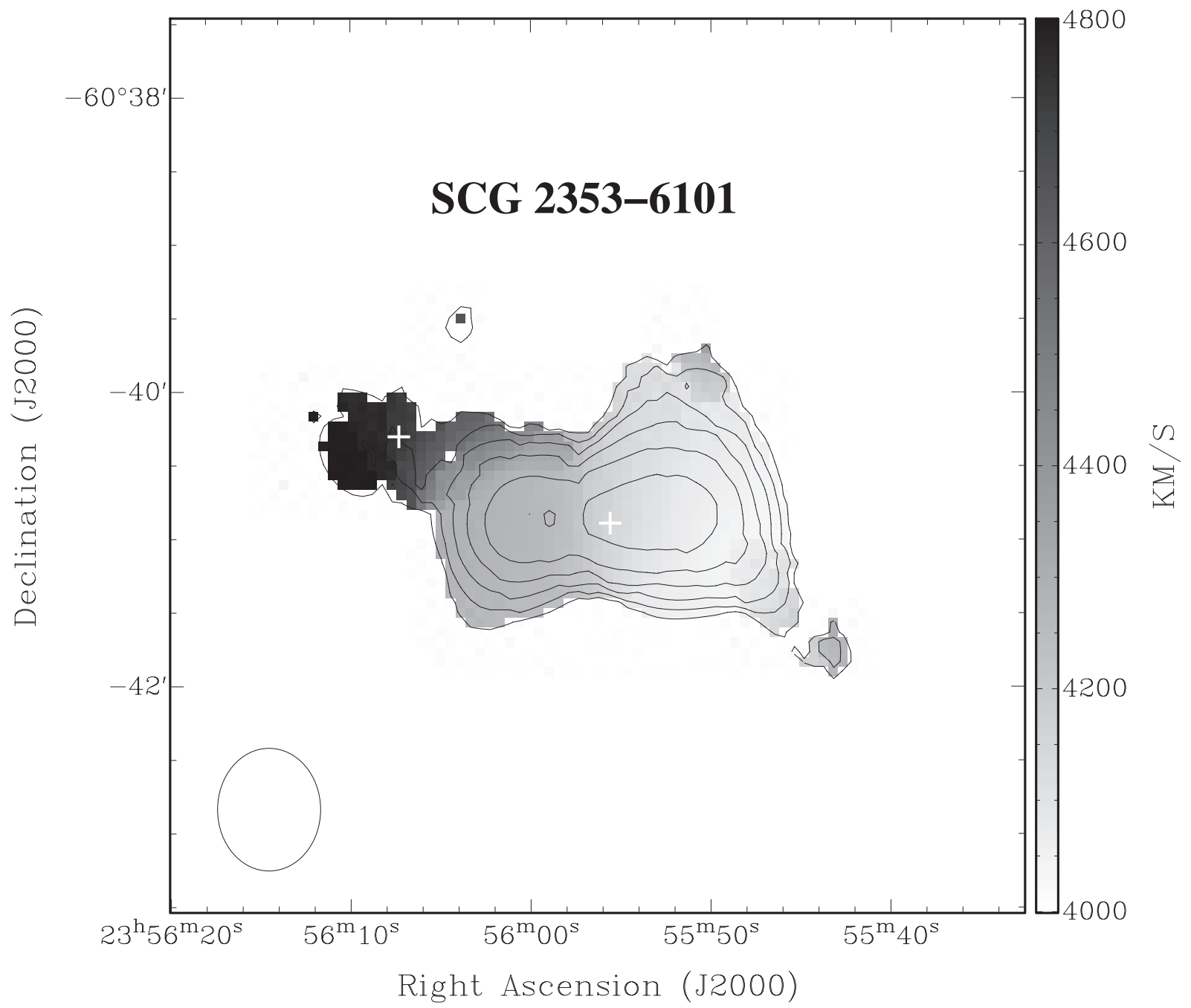

Fig. 4. ATCA H I image of SCG 2353-6101 superimposed on the H I velocity field, the white crosses indicate the center of galaxy $a$ and $b$. The contours are the same as in Fig. 3, the field of view is $5^{\prime} .9 \times 6^{\prime} 1$ and the angular resolution $\left(F W H M=50^{\prime \prime} \times 42^{\prime \prime}\right)$ is indicated in the lower left. The velocity scale is shown on the right and the orientation is $\mathrm{N}$ up and $\mathrm{E}$ to the left. 
E. Pompei et al.: Optical and radio study of six southern compact groups, Online Material p 13

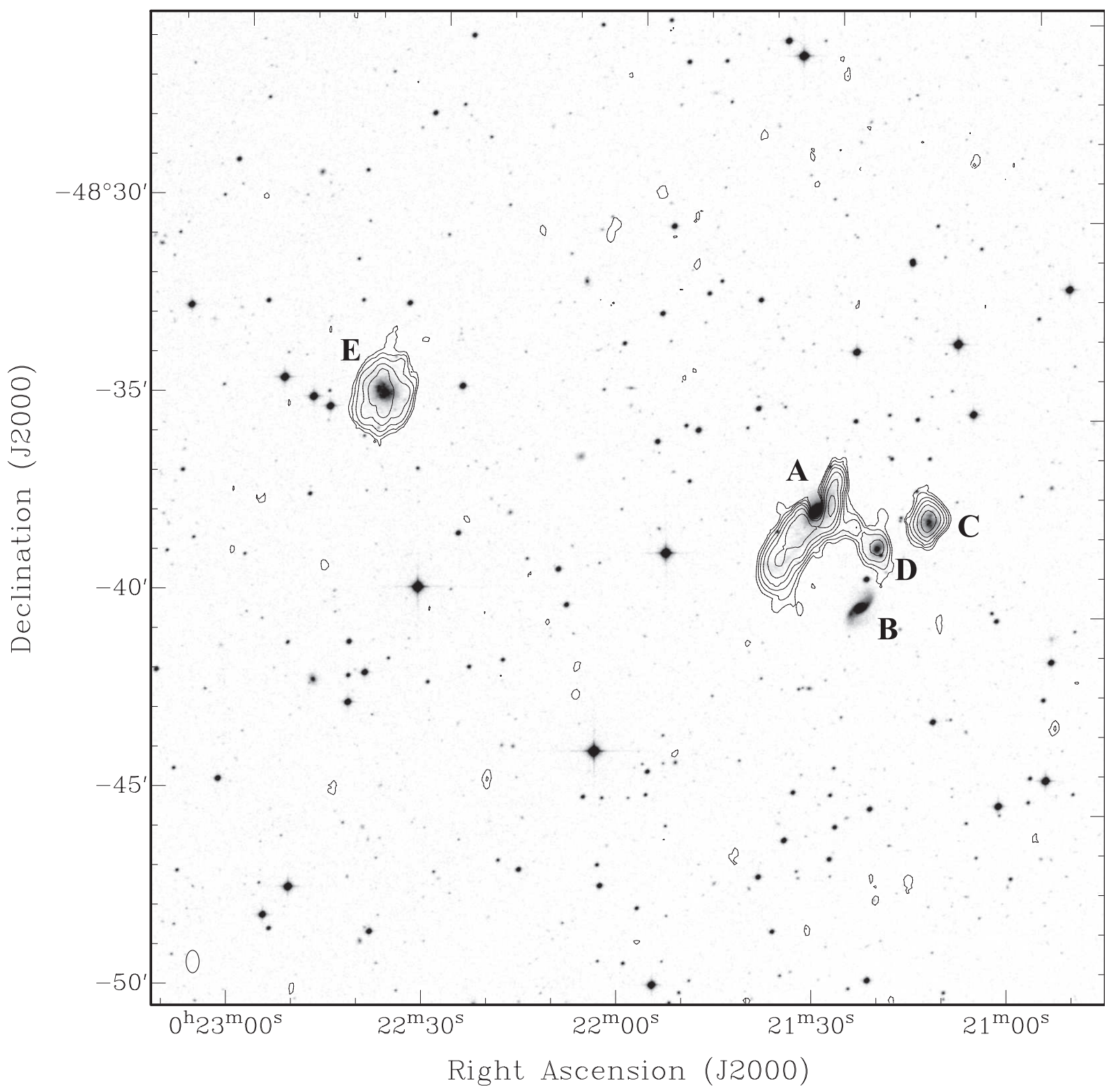

Fig. 5. ATCA H I image of SCG 0018-4854, superimposed on a red DSS-2 optical plate. The contour levels are at 0.12, 0.17, 0.24, 0.34, 0.48, 0.68 and $0.96 \mathrm{Jy} \mathrm{km} \mathrm{s}^{-1}$ beam $^{-1}$. The field of view is $24.2 \times 25.2$ and the angular resolution $\left(F W H M=38^{\prime \prime} \times 22^{\prime \prime}\right)$ is indicated in the lower left. $\mathrm{N}$ is up and $\mathrm{E}$ to the left. 
E. Pompei et al.: Optical and radio study of six southern compact groups, Online Material p 14

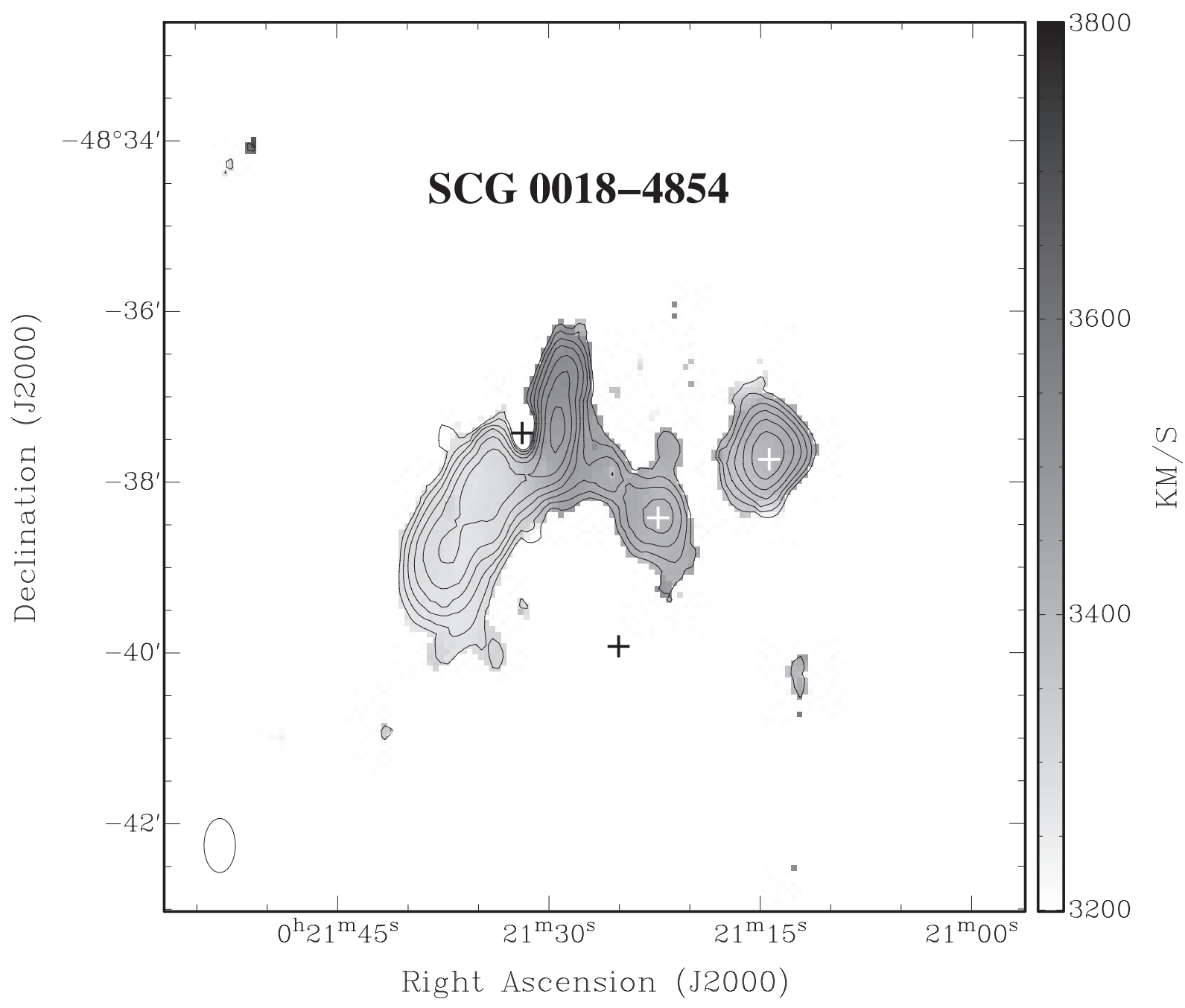

Fig. 6. ATCA H I image of SCG 0018-4854, superimposed on the H I velocity field. The contours are the same as in Fig. 5, the field of view is $10^{\prime} 2 \times 10^{\prime} 5$ and the angular resolution $\left(F W H M=38^{\prime \prime} \times 22^{\prime \prime}\right)$ is indicated in the lower left. Crosses indicate the center of each member galaxy, the velocity scale is shown on the right, and the orientation is $\mathrm{N}$ up and $\mathrm{E}$ to the left. 
E. Pompei et al.: Optical and radio study of six southern compact groups, Online Material p 15

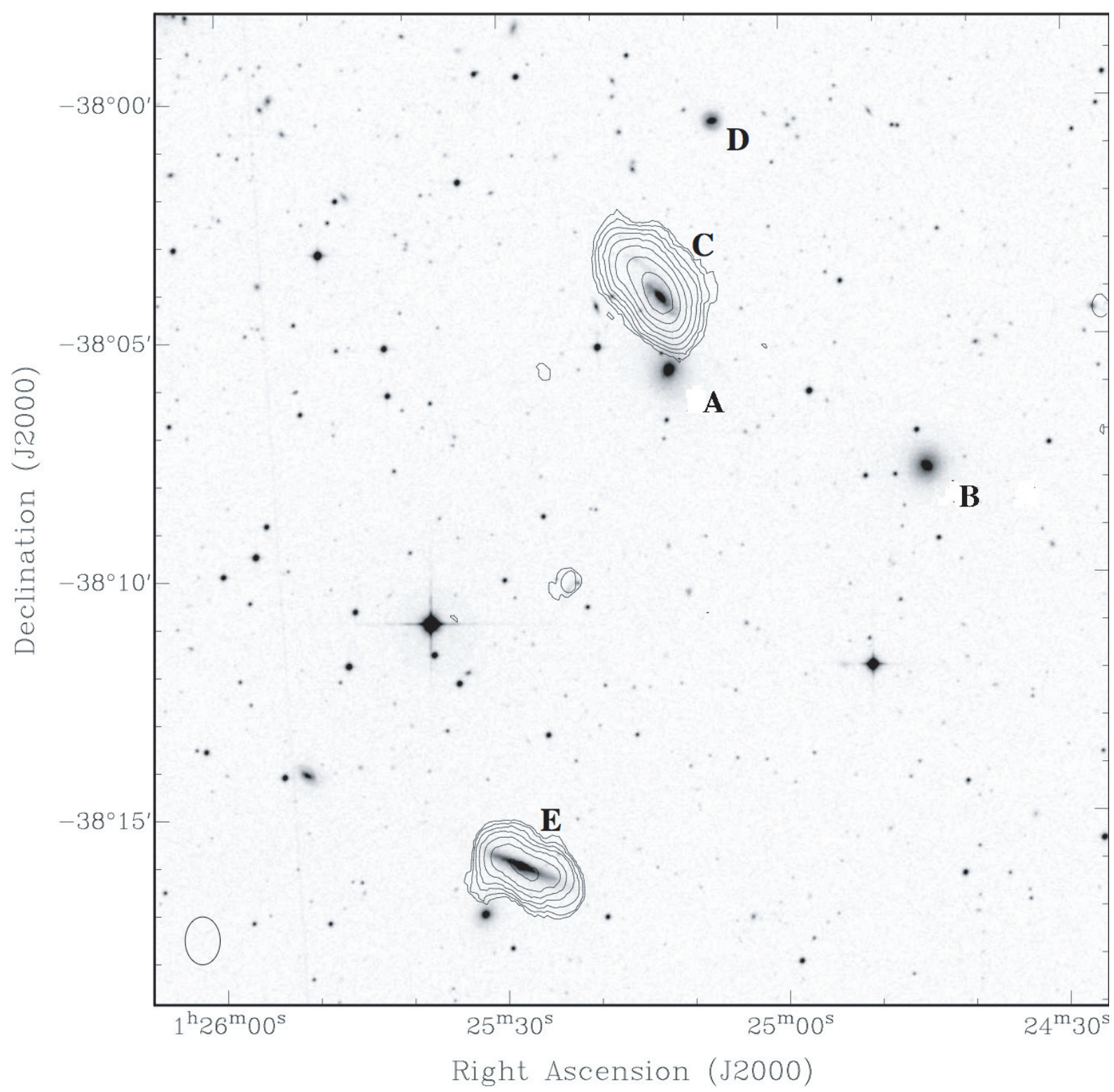

Fig. 7. ATCA H I image of SCG0122-3819, superimposed on a red DSS-2 optical plate. The contour levels are at 0.20 .280 .40 .560 .81 .121 .6 $2.24 \mathrm{Jy} \mathrm{km} \mathrm{s}^{-1}$ beam $^{-1}$. The field of view is $20^{\prime} 2 \times 20^{\prime} .9$ and the angular resolution $\left(F W H M=38^{\prime \prime} \times 22^{\prime \prime}\right)$ is indicated in the lower left. N is up and E to the left. 
E. Pompei et al.: Optical and radio study of six southern compact groups, Online Material p 16

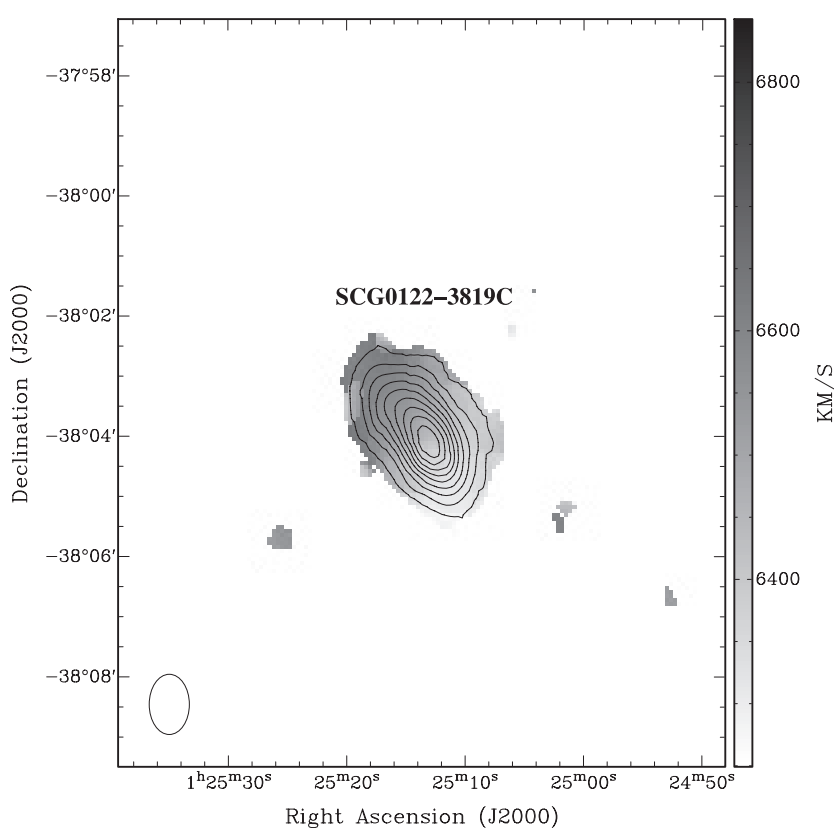

(a)

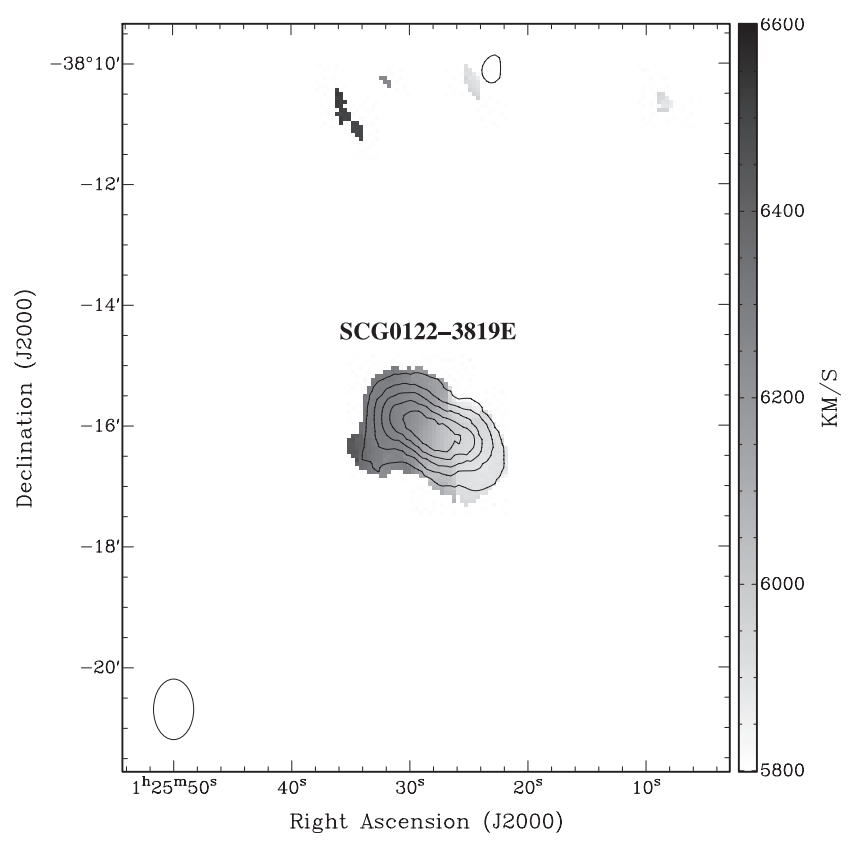

(b)

Fig. 8. ATCA H I image of SCG 0122-3819C (left panel) and E (right panel) superimposed on the H I velocity field. The contours are at the same levels as in Fig. 7; the field of view is 9.6 $\times 10^{\prime} .0$ and the angular resolution $\left(F W H M=60^{\prime \prime} \times 40^{\prime \prime}\right)$ is indicated in the lower left. The velocity scale is shown on the right, and the orientation of the image is $\mathrm{N}$ up and $\mathrm{E}$ to the left. 
E. Pompei et al.: Optical and radio study of six southern compact groups, Online Material p 17

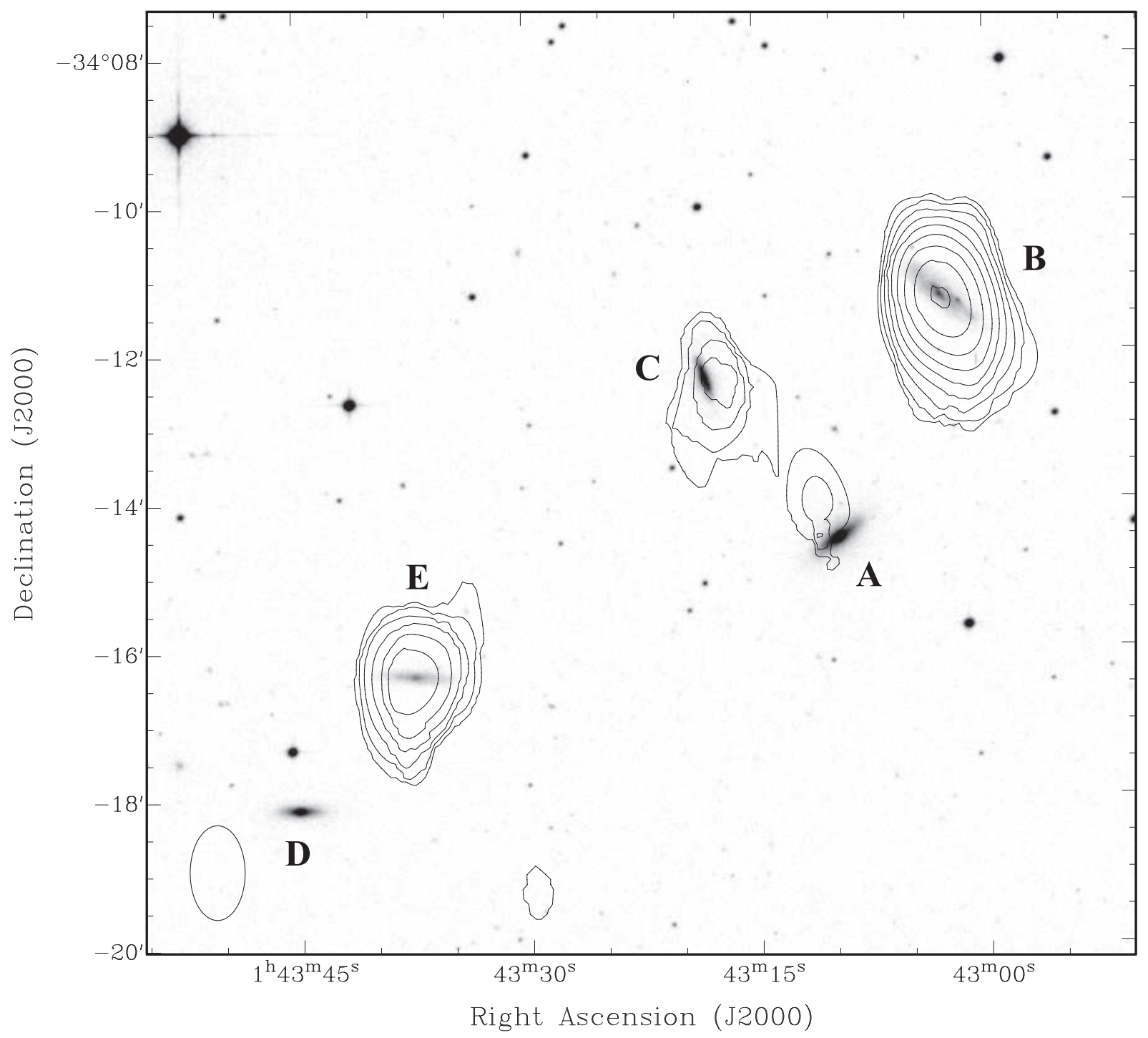

Fig. 9. ATCA H I image of SCG 0141-3429, superimposed on a red DSS-2 optical plate. The contour levels are at 0.170 .240 .340 .480 .680 .96 $1.361 .922 .6 \mathrm{Jy} \mathrm{km} \mathrm{s}^{-1}$ beam $^{-1}$. The field of view is $13.4 \times 12$ '.7 and the angular resolution $\left(F W H M=80^{\prime \prime} \times 44^{\prime \prime}\right)$ is indicated in the lower left. N is up and $\mathrm{E}$ to the left. 


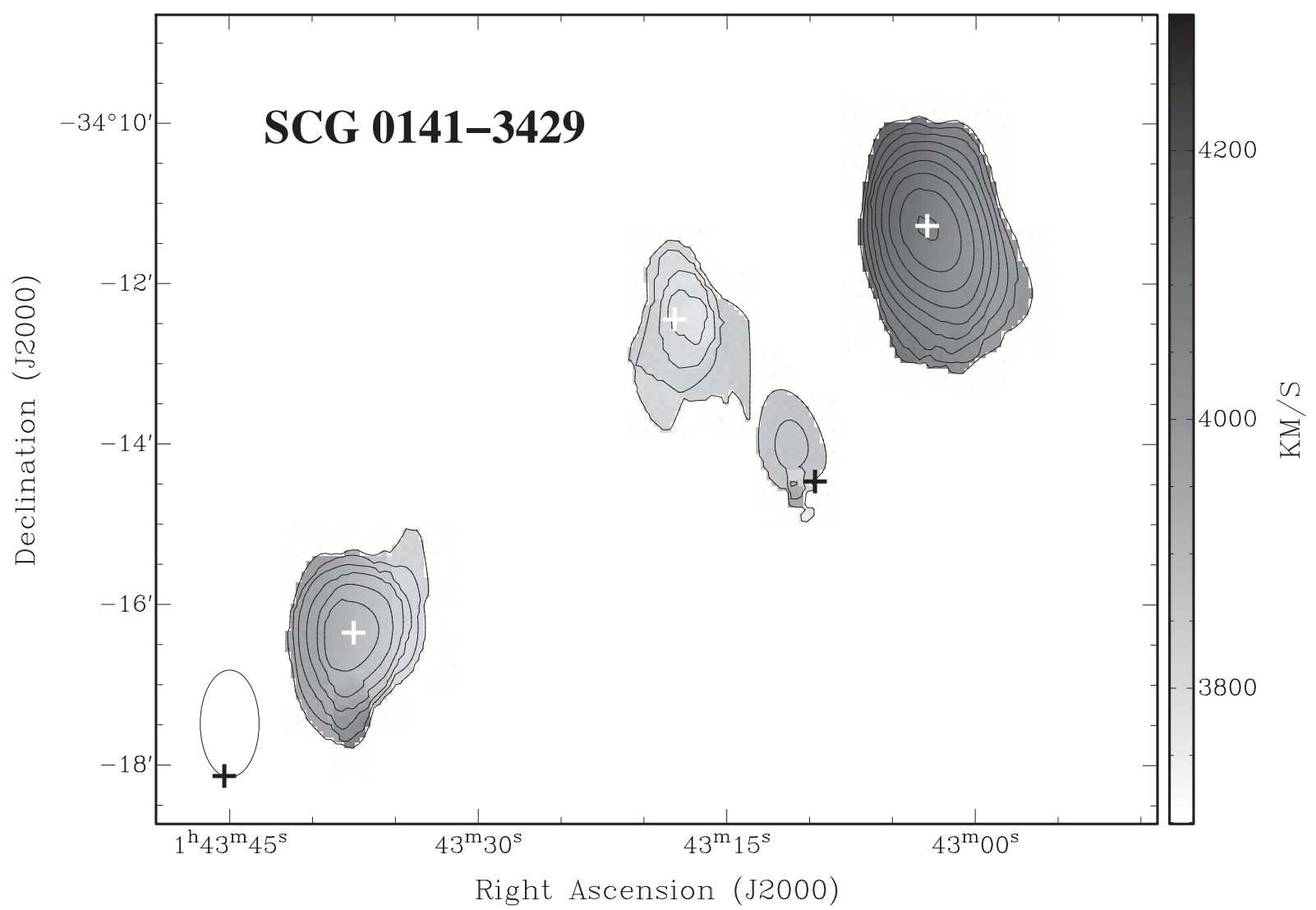

Fig. 10. ATCA H I contours of SCG 0141-3429 superimposed on the H I velocity field; the crosses indicate the centers of the member galaxies. The contours are the same as in Fig. 9, the field of view is $12.6 \times 10^{\prime} 1$ and the angular resolution $\left(F W H M=38^{\prime \prime} \times 22^{\prime \prime}\right)$ is indicated in the lower left. The velocity scale is shown on the right and the orientation is $\mathrm{N}$ up and $\mathrm{E}$ to the left. 
E. Pompei et al.: Optical and radio study of six southern compact groups, Online Material p 19

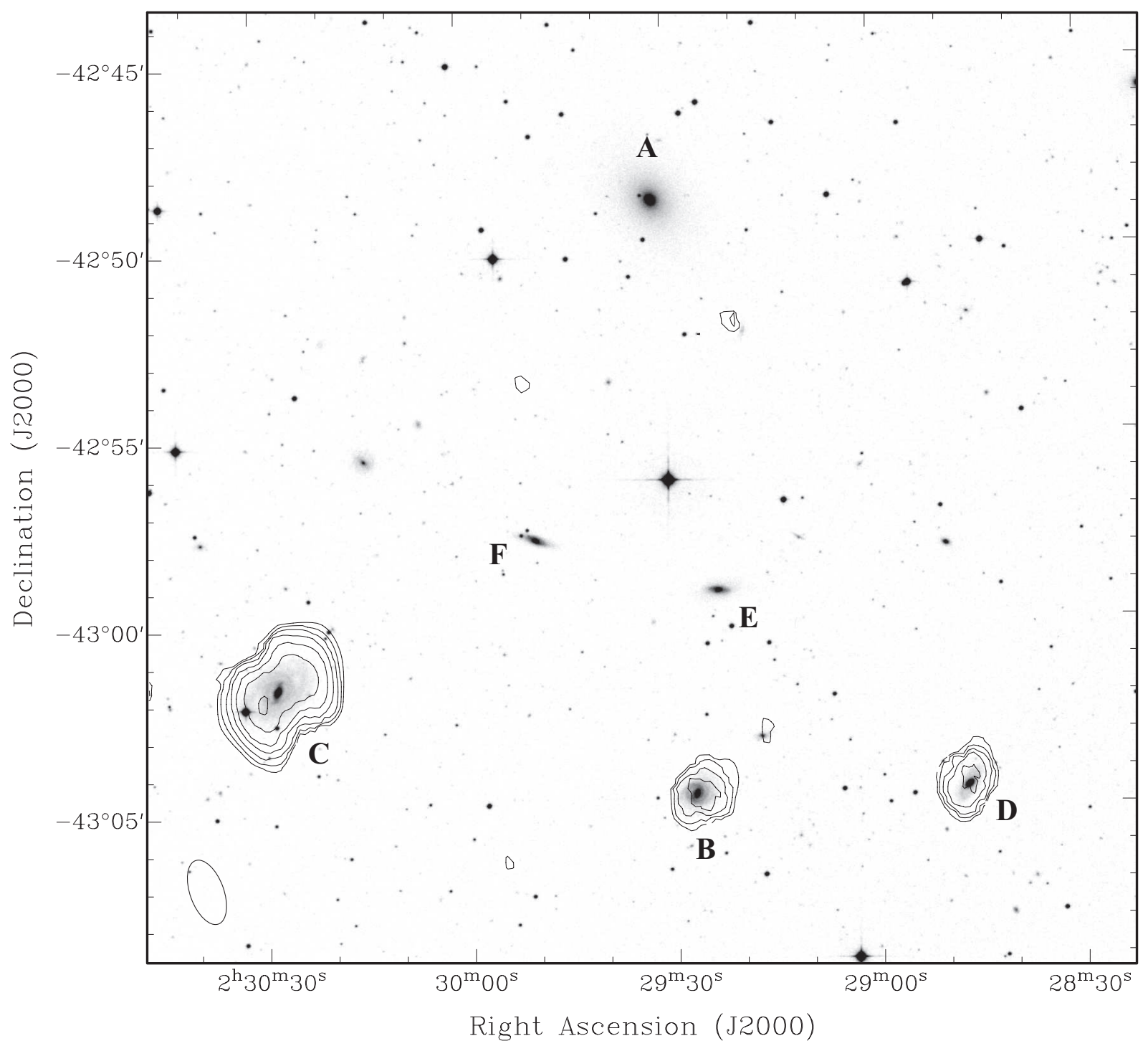

Fig. 11. ATCA H I image of SCG0227-4312, superimposed on a red DSS-2 optical plate. The contour levels are at 0.20 .280 .40 .560 .81 .121 .6 $2.24 \mathrm{Jy} \mathrm{km} \mathrm{s}^{-1}$ beam $^{-1}$. The field of view is $26^{\prime} 6 \times 25^{\prime} .4$ and the angular resolution $\left(F W H M=38^{\prime \prime} \times 22^{\prime \prime}\right)$ is indicated in the lower left. $\mathrm{N}$ is up and E to the left. 
E. Pompei et al.: Optical and radio study of six southern compact groups, Online Material $p 20$

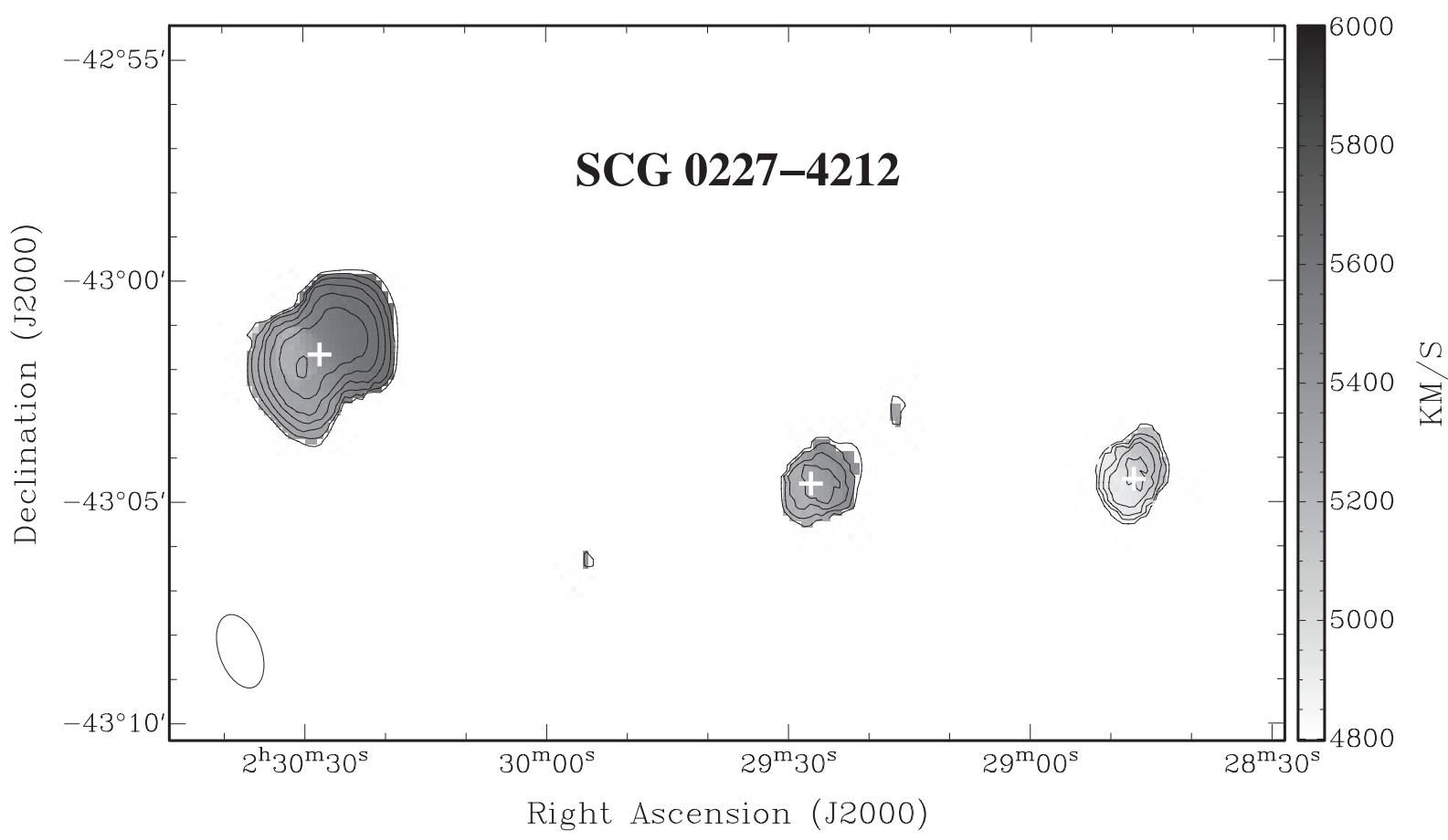

Fig. 12. ATCA H I image of SCG 0227-4212, superimposed on the H I velocity field. The contours are the same as in Fig. 11, the field of view is $23.5 \times 16^{\prime} 2$ and the angular resolution $\left(F W H M=104^{\prime \prime} \times 57^{\prime \prime}\right)$ is indicated in the lower left. The velocity scale is shown on the right and the orientation is $\mathrm{N}$ up and $\mathrm{E}$ to the left. 
E. Pompei et al.: Optical and radio study of six southern compact groups, Online Material p 21

Table 3 shows the result from the GALFIT analysis on the member galaxies of the six groups presented in the paper.

Table 3. Results from the GALFIT analysis on the member galaxies of our SCGs. The symbols used here have the same meaning as in Peng 2002; the position angle is measured from $\mathrm{N}$ to $\mathrm{E}$ counterclockwise. The magnitude is the total magnitude of the component, corrected for galactic extinction; errors on the measured magnitudes are of the order of 0.05 magnitudes and refer to the accuracy in the measurement of the zero-point plus the sky subtraction and the statistical error from GALFIT. Group SCG2159-3210 and galaxy SCG0122-3819e have not been observed by us in the optical; for photometric measurement the reader is referred to Hickson et al. 1982 and to NED.

\begin{tabular}{|c|c|c|c|c|c|c|c|c|}
\hline Name of the galaxy & Functional form & $\begin{array}{l}\text { Mag } \\
\text { (R) }\end{array}$ & $\begin{array}{c}r_{\mathrm{e}, \mathrm{s}}{ }^{a} \\
(\operatorname{arcsec})\end{array}$ & $n$ & $q$ & $\begin{array}{c}\text { PA } \\
(\mathrm{deg})\end{array}$ & $c^{b}$ & Comments $^{c}$ \\
\hline \multirow[t]{3}{*}{ SCG 2353-6101A } & Sersic & 15.14 & 2.14 & 0.76 & 0.79 & -59.1 & -0.01 & (R'_1)SAB(rs)b, SFG \\
\hline & Sersic & 15.42 & 10.46 & 0.5 & 0.29 & -75.2 & - & central source, point-like \\
\hline & Expdisk & 13.26 & 10.21 & - & 0.71 & -70.8 & -0.18 & strong, twisted spiral arms \\
\hline \multirow[t]{2}{*}{ SCG 2353-6101B } & Sersic & 16.24 & 11.59 & 1.29 & 0.85 & 68.9 & -0.45 & Scpec, LINER \\
\hline & Expdisk & 13.52 & 14.33 & - & 0.34 & 87.8 & -0.13 & very diffuse spiral arms \\
\hline \multirow[t]{3}{*}{ SCG 2353-6101C } & Sersic & 14.27 & 16.18 & 2.11 & 0.81 & 79.0 & 0.08 & So \\
\hline & Sersic & 14.8 & 1.4 & 0.64 & 0.98 & 83.6 & - & central source, point-like \\
\hline & Expdisk & 13.74 & 3.96 & - & 0.42 & 83.1 & -0.16 & \\
\hline \multirow[t]{3}{*}{ SCG 0018-4854A } & Sersic & 13.68 & 6.20 & 1.99 & 1.00 & 80.3 & -0.7 & Sa $a p$ \\
\hline & Sersic & 12.76 & 31.18 & 2.26 & 0.42 & -22.8 & -0.28 & \\
\hline & Expdisk & 13.13 & 4.94 & - & 0.62 & -32.7 & -0.17 & extended tidal tail \\
\hline SCG 0018-4854B & Expdisk & 12.72 & 6.66 & - & 0.52 & -48.9 & -0.05 & SB0/apec, Sy2 \\
\hline SCG 0018-4854C & Expdisk & 13.66 & 7.79 & - & 0.79 & 5.4 & -0.49 & Irr, SFG \\
\hline \multirow[t]{2}{*}{ SCG 0018-4854D } & Sersic & 15.90 & 2.01 & 1.00 & 0.92 & -17.0 & 0.21 & SBabpec, LINER \\
\hline & Expdisk & 14.01 & 5.91 & - & 0.75 & -21.0 & 0.35 & \\
\hline \multirow[t]{2}{*}{ SCG 0018-4854E } & Sersic & 15.90 & 2.01 & 1.00 & 0.92 & -17.0 & 0.21 & $\mathrm{SAB}(\mathrm{r}) \mathrm{c}, \mathrm{SFG}$ \\
\hline & Expdisk & 14.01 & 5.91 & - & 0.75 & -21.0 & 0.35 & \\
\hline \multirow[t]{3}{*}{ SCG 0122-3819A } & Sersic & 13.61 & 3.66 & 1.19 & 0.85 & -23.7 & 0.03 & $\mathrm{SBa}$ \\
\hline & Bar & 14.10 & 42.3 & - & 0.77 & -30.0 & 0.01 & flat profile bar \\
\hline & Expdisk & 13.26 & 15.2 & - & 0.37 & -56.1 & -0.29 & \\
\hline \multirow[t]{3}{*}{ SCG 0122-3819B } & DV & 13.72 & 22.3 & - & 0.42 & 39.2 & -0.15 & SB0, LLAGN \\
\hline & Bar & 14.10 & 42.3 & - & 0.77 & -30.0 & 0.01 & flat profile bar \\
\hline & Expdisk & 14.60 & 4.12 & - & 0.43 & 43.1 & -0.23 & strongly twisted spiral arms \\
\hline \multirow[t]{2}{*}{ SCG0122-3819C } & Sersic & 13.72 & 22.3 & 1.09 & 0.42 & 39.2 & -0.15 & $\mathrm{Sc}, \mathrm{SFG}$ \\
\hline & Expdisk & 14.60 & 4.12 & - & 0.43 & 43.1 & -0.23 & strongly twisted spiral arms \\
\hline \multirow[t]{2}{*}{ SCG 0122-3819D } & Sersic & 15.11 & 3.11 & 1.00 & 0.66 & -79.6 & -0.29 & SOB, exponential bar \\
\hline & Expdisk & 14.53 & 4.77 & - & 0.99 & -78.9 & 0.17 & \\
\hline \multirow[t]{2}{*}{ SCG 0141-3429A } & Sersic & 14.12 & 1.97 & 0.79 & 0.61 & -58.8 & -0.01 & $\mathrm{~S}^{+}$ \\
\hline & Expdisk & 13.11 & 8.00 & - & 0.37 & -56.1 & -0.29 & \\
\hline \multirow[t]{2}{*}{ SCG 0141-3429B } & Sersic & 16.60 & 4.31 & 0.65 & 0.33 & 32.7 & -0.59 & $\mathrm{SAB}(\mathrm{s}) \mathrm{bcpec}$ \\
\hline & Sersic & 13.98 & 24.44 & 0.5 & 0.27 & 49.5 & 0.02 & \\
\hline \multirow[t]{2}{*}{ SCG 0141-3429C } & Sersic & 14.29 & 10.40 & 0.51 & 0.21 & 17.5 & -0.13 & $\mathrm{SB}(\mathrm{rs}) 0, \mathrm{SFG}$ \\
\hline & Expdisk & 15.11 & 6.41 & - & 0.66 & 17.3 & 0.83 & \\
\hline \multirow[t]{2}{*}{ SCG 0141-3429D } & Sersic & 15.83 & 1.34 & 0.76 & 0.88 & 88.3 & - & So \\
\hline & Sersic & 14.07 & 9.55 & 1.29 & 0.29 & 87.9 & -0.20 & \\
\hline SCG 0141-3429E & Sersic & 14.92 & 19.22 & 0.93 & 0.21 & 84.5 & -0.40 & $\mathrm{Sc} s p, \mathrm{SFG}$ \\
\hline \multirow[t]{2}{*}{ SCG 0227-4312A } & Sersic & 11.55 & 42.81 & 2.95 & 0.79 & 34.8 & 0.09 & So \\
\hline & Sersic & 13.97 & 2.59 & 0.97 & 0.92 & 31.9 & -0.04 & \\
\hline \multirow[t]{2}{*}{ SCG 0227-4312B } & Sersic & 14.42 & 5.11 & 1.37 & 0.66 & -15.7 & -0.16 & (R)SAB(rs)b, EMLG \\
\hline & Sersic & 13.18 & 17.05 & 0.53 & 0.89 & -64.0 & 0.09 & \\
\hline \multirow[t]{3}{*}{ SCG 0227-4312C } & Sersic & 14.76 & 10.97 & 0.50 & 0.31 & -16.1 & -0.33 & $\mathrm{Sbc}$ \\
\hline & Sersic & 14.20 & 3.07 & 0.90 & 0.79 & -40.8 & -0.06 & \\
\hline & Expdisk & 12.29 & 27.60 & - & 0.65 & -52.0 & -0.12 & \\
\hline \multirow[t]{2}{*}{ SCG 0227-4312D } & Sersic & 13.79 & 15.22 & 1.49 & 0.44 & -40.8 & -0.27 & $\mathrm{SBb}$, LLAGN \\
\hline & Sersic & 14.42 & 3.54 & 1.00 & 1.00 & 8.8 & -0.58 & \\
\hline \multirow[t]{3}{*}{ SCG 0227-4312E } & Sersic & 14.87 & 16.37 & 0.68 & 0.25 & 89.9 & 0.73 & S0, SFG \\
\hline & Sersic & 14.37 & 7.65 & 2.02 & 0.83 & -89.4 & 0.85 & \\
\hline & Expdisk & 16.11 & 7.32 & - & 0.25 & 76.4 & 0.79 & \\
\hline SCG 0227-4312F & Sersic & 15.40 & 3.67 & 1.05 & 1.00 & 68.9 & - & $\mathrm{Sa}$ \\
\hline & Sersic & 15.12 & 13.44 & 1.00 & 0.31 & 58.2 & 0.20 & \\
\hline & Expdisk & 14.34 & 11.33 & - & 0.24 & 71.7 & 0.19 & \\
\hline
\end{tabular}

${ }^{a} r_{\mathrm{e}}$ is the effective radius of the Sersic or the deVaucouleurs's law, while $\mathrm{r}_{s}$ is the scale length of the exponential disk.

${ }^{b}$ Diskiness (negative) or boxiness (positive) paramenter. It has no real meaning for central sources, so it is not reported for these cases.

${ }^{c}$ Activity classification for HCG 90 is given in Coziol et al. (1998), while for all other groups, except SCG0141-3429, is from Coziol et al. (2000). 